\title{
Translational Cardiovascular Modeling: Tetralogy of Fallot and Modeling of Diseases
}

\author{
Radomír Chabiniok, Kateřina Škardová, Radek Galabov, Pavel Eichler, Maria \\ Gusseva, Jan Janoušek, Radek Fučík, Jaroslav Tintěra, Tomáš Oberhuber, Tarique \\ Hussain
}

\author{
Abstract Translational cardiovascular modeling (TCM) combines clinical data with \\ physiologically and biophysically based models of the heart, vessels or circulation, \\ while aiming to contribute to diagnosis or optimal clinical management. Models \\ of heart mechanics and electromechanical models are applicable when assessing \\ ventricular function, contributing to planning of optimal intervention. During a pe- \\ rioperative period or acute exacerbation of heart failure, close to real time running \\ models can be coupled with signals monitoring cardiovascular physiology. Blood \\ flow assessed by combining phase contrast magnetic resonance imaging with flow \\ Radomír Chabiniok \\ UT Southwestern Medical Center Dallas, TX, USA \\ Inria France \\ Ecole Polytechnique, CNRS, Institut Polytechnique de Paris, Palaiseau, France \\ St. Thomas' Hospital, King's College London, UK \\ Faculty of Nuclear Sciences and Physical Engineering, Czech Technical University in Prague, \\ Czech Republic \\ e-mail: radomir.chabiniok@utsouthwestern.edu \\ Kateřina Škardová, Pavel Eichler, Radek Fučík, Tomáš Oberhuber \\ Faculty of Nuclear Sciences and Physical Engineering, Czech Technical University in Prague, \\ Czech Republic \\ Radek Galabov, Jaroslav Tintěra \\ Institute for Clinical and Experimental Medicine in Prague, Czech Republic \\ Maria Gusseva \\ Inria France \\ Ecole Polytechnique, CNRS, Institut Polytechnique de Paris, Palaiseau, France \\ Jan Janoušek \\ Children's Heart Center, University Hospital Motol, Charles University, Prague, Czech Republic \\ Tarique Hussain \\ UT Southwestern Medical Center Dallas, TX, USA
}


models can contribute to the decision about a possible intervention e.g. on heart valves or large vessels. Furthermore, advanced imaging and image processing constrained by biophysical models allows for the study of distinct patterns, which could contribute to early detection or mapping a disease progress. In this chapter we demonstrate applicability of some TCM methods on tetralogy of Fallot (TOF) the most common cyanotic congenital heart disease. A number of already existing modeling techniques can be applied on the cohort of TOF. Likewise, some novel techniques developed specifically for the group of TOF patients could serve in some other pathologies. This whole approach leads to an acronym TOFMOD, standing for Tetralogy of Fallot and Modeling of Diseases. 


\section{Introduction}

Translational cardiovascular modeling (TCM) plays a crucial role in extending highfidelity engineering models into real-life clinical applications. It represents a novel multi-disciplinary approach, which brings the opportunity to address clinical problems not sufficiently solved by current techniques and contributes into advancing healthcare of pathologies involving cardiovascular system. As reviewed in [1], the components of TCM are: (1) physiologically and biophysically based models of the heart, flow in vessels or circulation; (2) acquisition and processing pipelines for clinical data; (3) means of creation of personalized models (i.e. model-data fusion); and (4) translation of patient-specific modeling into patient care, contributing to diagnosis or optimal clinical management.

We will present TCM through the application into one particular disease named tetralogy of Fallot (TOF). TOF is a disease, for which several TCM techniques can be combined: for instance, assessment of myocardial functional state can be performed by employing models of heart mechanics; planning of cardiac resynchronization therapy can be assisted by using electromechanical heart models; advanced imaging and image processing constrained by biophysical models allow to study distinct patterns in TOF patients; and assessment of narrowing of a pulmonary artery branch and possible indication for an intervention (e.g. stenting) can be facilitated by using large-vessel flow models. This chapter presents some of these techniques.

\subsection{Tetralogy of Fallot}

Tetralogy of Fallot is a common congenital heart disease. It accounts for 7-10\% of all congenital heart diseases. If untreated, it would lead to death in infancy. During the embryologic phase, the aorticopulmonary septum (dividing the cardiac outflow channel into aortic and pulmonary outflows [2]) is deviated anteriorly causing the two outflow arteries - pulmonary artery (PA) and aorta (from the right and left ventricle, respectively) - to be of different size (narrower PA and wider proximal aorta). As depicted in Fig. 1(b), this causes (1) narrowing of the right ventricular outflow tract (RVOT) and PA; (2) the aorta overriding both left and right ventricular outflow tracts (therefore blood from both ventricles can enter the aorta); (3) ventricular septal defect (VSD); and (4) hypertrophy of the right ventricle (RV), which compensates the RV working against high afterload (due to the stenosed RVOT). Additionally, TOF patients often suffer from other associated defects, out of which stenosis of a branch of the PA (left or right pulmonary artery, LPA, RPA) is common. Mixing of oxygenated and deoxygenated blood entering the aorta from the LV and RV, respectively, causes a typical bluish color of the skin, the so-called cyanosis, and TOF represents the most common cyanotic congenital heart disease. The four classical symptoms of this blue baby syndrome (la maladie bleue) were described by the French physician Etienne-Louis Arthur Fallot in 1888 [3]. 


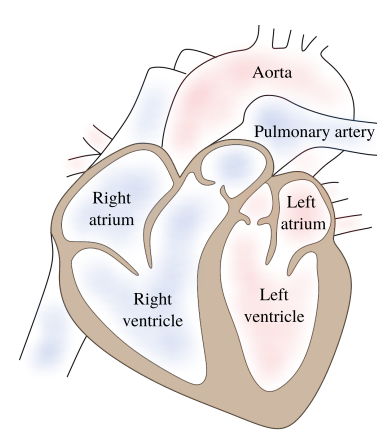

(a) Normal heart

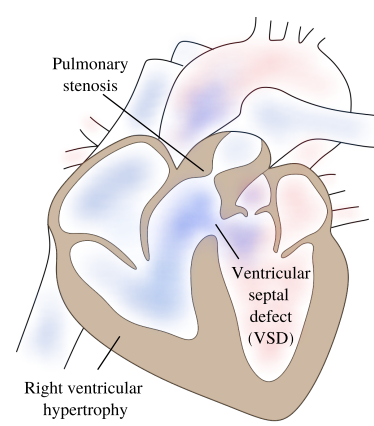

(b) Tetralogy of Fallot (TOF)

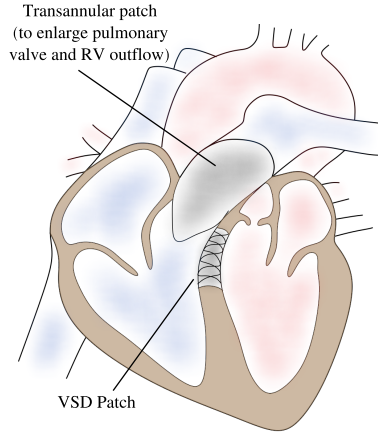

(c) Complete surgical repair (rTOF)

Fig. 1 Schematics of normal anatomy of heart and large vessels and of tetralogy of Fallot.

Complete surgical repair of TOF (rTOF) is usually performed during the first year of life. It consists of repairing the VSD and increasing the size of the RVOT and PA, as depicted in Fig. 1.(c). The correction of the PA stenosis (causing the initial pressure RV overload and RV hypertrophy), often made by using a transannular patch, typically disrupts the function of the pulmonary valve and causes a pulmonary regurgitation (PR), i.e. "leaking pulmonary valve". RV is then chronically volume overloaded. Moreover, even after the repair of tetralogy of Fallot, some rTOF patients suffer from a residual stenosis of the RV outflow tract (RVOT). This eventually leads to a combined RV volume and pressure overload. The chronic volume overload causes $\mathrm{RV}$ remodeling in the sense of RV dilatation and the chronic pressure overload leads to the hypertrophy of the RV myocardium [4]. The hearts of rTOF patients often have electrical activation abnormalities either as a consequence of the surgical repair or developed in the remodeled heart. The right bundle branch block (RBBB) - i.e. a block in the main electrical conduction pathway, which normally allows a rapid transmission of electrical activation throughout RV [5] - is common. It causes a dyssynchrony in the activation and contraction (a delayed activation and contraction of the RV free wall), which decreases cardiac efficiency [6]. Malignant ventricular arrhythmias (e.g. ventricular fibrillation) lead to sudden cardiac death and are the most common cause of death after the repair of TOF [7].

To avoid uncontrolled RV remodeling and even to allow the RV to reverse remodel back to normal size, the patients undergo later in life a pulmonary valve replacement (PVR). PVR would ideally be performed as late as possible (intervention needs to be repeated throughout life as the lifespan of the implanted valve is 5-10 years [8]), but before irreversible changes of the myocardium or before a significant electrophysiology event takes place [9, 10, 11]. The optimal timing is crucial both for the initial PVR as well as for the subsequent replacements of the implanted valve [12, 13].

Additionally to the RV-related issues, rTOF patients are known to suffer from LV failure earlier than in normal population. The reason for the LV involvement in TOF, 
which is classically considered as a "right heart disease", is not entirely clear. Both mechanical (e.g. dyssynchrony in contraction) as well as humoral factors (which can lead e.g. to an increased tissue stiffness of both ventricles [14]) may be involved and modeling has the potential to shed light on some of them.

While the complete surgical repair increases the life expectancy from a few years to many decades up to normal life expectancy [15], the rTOF patients must undergo regular follow up exams and their clinical management is still facing limits for example in:

1. Optimal timing of PVR

2. Optimal indication and timing of stenting of a stenosed branch pulmonary artery

3. Optimal management of electrophysiological (EP) disorders including atrial arrhythmias, ventricular arrhythmias and prevention of sudden cardiac death

4. Preventing left- and right-sided heart failure

These items represent some of the challenges of current clinical care. In next sections, we demonstrate some paths in which TCM can contribute to clinical management of rTOF patients. In Section 2 we show how can TCM be applied during assessing ventricular function either at regular follow up exams when detailed clinical data are available (Secs. 2.1 and 2.2), or in a perioperative period or acute exacerbation of heart failure, while coupling fast running models with continuously acquired signals monitoring cardiovascular physiology at operation theatre or intensive care unit (ICU, Sec.2.3). Section 2.4demonstrates some paths of including the models in the assessment of a long-term cardiac performance. Section 3 presents the pulmonary right ventricular resynchronization in congenital heart diseases, as for rTOF. Physiological and physical knowledge, the so-called "model a priori", can be incorporated into advanced imaging and image processing techniques. Section 4 presents some examples in which model-constrained image processing allowed to study some distinct patterns of TOF. Those could represent initial signs of earlystage heart failure or be associated with a disease progress. Section 5 shows the steps towards coupling flow models with clinical data to overcome some physical limits of the imaging techniques. Section 6 further discusses various aspects of the clinical modeling for rTOF patients and shows some connections between this particular congenital heart disease and models for general population and acquired heart diseases. Section 7 concludes this chapter.

\section{Ventricular mechanics and its biomechanical modeling}

Coupling image data with biophysical models allows to estimate the biophysical properties of the heart and vascular system. Such quantities are not directly measurable from the image and pressure data but are accessible via constitutive relations and equilibrium equations (see reviews [16, 1] and references therein). Moreover, the physical character predisposes the biophysical models to predictivity. In this section, 
we provide a more detailed look on some of these techniques which can be applied in rTOF patients.

\subsection{Assessment of right ventricular mechanics in rTOF patients}

In Section 1.1 we explained that rTOF patients suffer from a chronic PR or RVOT obstruction leading to a volume or pressure overload of their RV. The patients undergo PVR and the procedure needs to be repeated approximately every 10 years. It is therefore of utmost interest to predict when the pathological remodeling of $\mathrm{RV}$ is approaching an irreversible state and suggest to perform the intervention immediately, or defer it and suggest a suitable timing for the next follow up exam.

While the current works and clinical guidelines focus on the direct measures taken from image data (typically ventricular volumes and transvalvular flow, performed by imaging techniques), the underlying mechanism of the chronically overloaded tissue undergoing pathological remodeling is unknown.

Gusseva et al. hypothesized in [17] that accessing directly mechanical properties of the heart in rTOF patients will provide additional metrics which may better stratify the PVR-responders vs. non-responders. A biomechanical model of single heart cavity developed by Caruel et al. [18] was used. The preload was imposed from the measured pressure data in diastole. The heart model was connected to a Windkessel model of circulatory system (lumped-parameter model representing the circulation by a combination of resistive and capacitive elements in the analogy to electrical circuit [19]). The schematics of the model is shown in Fig. 22a). The geometry and kinematics of the ventricle are reduced to a sphere with the inner radius $R$ and wall thickness $d$. The constitutive mechanical laws were preserved as in the full 3D heart model described in [20] and [21]. The model was adjusted into a patient-specific regime. This allowed to plot RV pressure-volume (PV) loops for every patient pre- / post-PVR (see example on Fig. 2(b)). In addition, the patientspecific models allowed to access the parameter of RV contractility, i.e. the active stress developed in each sarcomere unit.

The myocardial contractilities prior to and after PVR for 20 patients included in study [17] are plotted in Fig. 3 (a) and the estimate of RV stroke work (an area encompassed within the PV loop) in Fig. 3 (b). Thanks to the valvular component (a system of diodes with forward and backward resistance, $R_{\text {for }}^{\mathrm{RVOT}}$ and $R_{\mathrm{back}}^{\mathrm{RVOT}}$, respectively, see Fig. 2(a)) the model allowed to study an independent effect of PR and RVOT obstruction on ventricular mechanics. Light blue and purple circles in Fig. 3 demonstrate pre- and post-PVR contractility (stroke work) for patients with predominantly high PR and RVOT obstruction, respectively. Fig. 3 3 shows a systematic trend of a decrease of RV contractility and stroke work observed immediately after deploying the valve. The lower inotropic (contractility) level would be more favorable with respect to the energy need of the myocardium, while the heart would be still providing an adequate cardiac output into the circulation (in fact, even 20-50\% higher than during the chronic PR, which is in line with experimental data by Lurz 


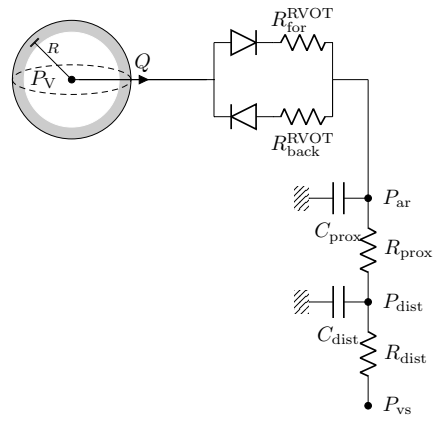

(a) Model schematics

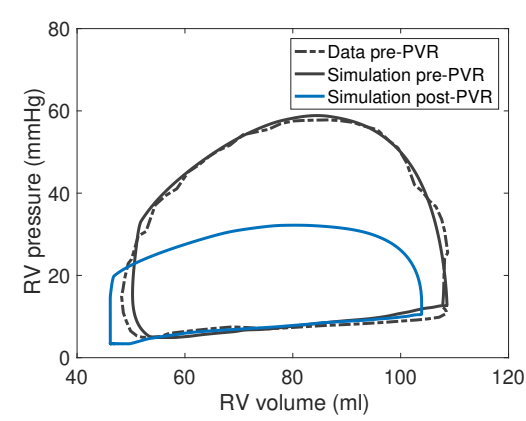

(b) Model-derived pressure-volume loops

Fig. 2 Schematics of the heart model coupled to the circulation system (a) and an example of model-derived pressure-volume loops prior to and after pulmonary valve replacement (PVR) (b). $Q$, outflow from right ventricle (RV); $R_{\text {for }}^{\text {RVOT }}$ and $R_{\text {back }}^{\text {RVOT }}$, forward and backward resistance of RV outflow tract; $R_{\text {prox }}, R_{\text {dist }}$, resistance of proximal, distal part of pulmonary circulation; $P_{\mathrm{V}}$, $P_{\mathrm{ar}}, P_{\mathrm{dist}}, P_{\mathrm{vs}}$, pressures in right ventricle, pulmonary artery, distal pulmonary circulation and pulmonary veins.

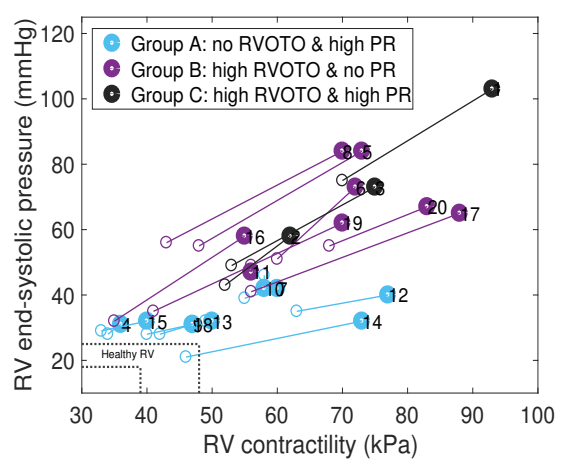

(a) Model-accessed contractility of RV

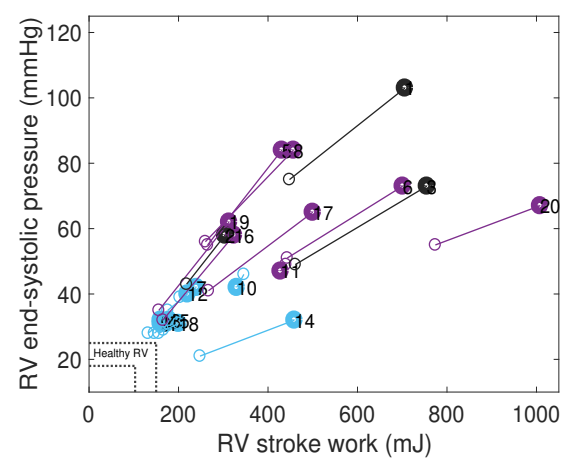

(b) RV stroke work

Fig. 3 Study estimating contractility and stroke work of right ventricles in 20 patients with repaired tetralogy of Fallot prior to (colored circles) and after pulmonary valve replacement (empty circles). PR: pulmonary regurgitation; RV: right ventricle; RVOTO: RV outflow tract obstruction.

et al. [22]). Moreover, the pre-PVR models revealed lower levels of contractility (and stroke work) in patients with predominant PR suggesting that ventricles are likely to adapt better to a long-term exposure to valvular regurgitation than to RVOT obstruction, which is also discussed in study [17].

This example shows that biomechanical modeling has the potential to contribute into personalized intervention by enhancing the ability to predict RV performance 
after PVR. This represents a way to baseline and track the RV performance over time, and allows for clinicians to make more informed decisions about PVR in patients with rTOF.

\subsection{Early-stage heart failure assessment}

Heart failure (HF) is the syndrome in which the heart is not able to pump a sufficient amount of blood to cover metabolic needs of tissues. The HF patients suffer from symptoms such as shortness of breath, cough, palpitations or dizziness. Early-stage $\mathrm{HF}$ is characterized by HF symptoms present only under more than ordinary physical activity (class I-II according to the New York Heart Association NYHA, see Table 1 . The assessment of early-stage $\mathrm{HF}$ therefore requests performing a clinical exam under physical exercise.

\begin{tabular}{|l|l|}
\hline Class & Objective Assessment \\
\hline I & $\begin{array}{l}\text { Patient with cardiac disease but without any limitations in physical activity } \\
\text { Comfortable at rest, ordinary physical activity results in fatigue, palpitations, } \\
\text { dyspnea or anginal pain }\end{array}$ \\
III & $\begin{array}{l}\text { Comfortable at rest, but less than ordinary physical activity results in fatigue, } \\
\text { palpitations, dyspnea or anginal pain } \\
\text { Symptoms of HF may be present even at rest }\end{array}$ \\
IV
\end{tabular}

Table 1 New York Heart Association classification for the heart failure (NYHA).

A decrease in exercise tolerance in rTOF patients is frequent. There are a number of possible causes: extracardiac (such as an increased RV afterload e.g. due to the RVOT obstruction, branch pulmonary artery stenosis, pulmonary pathology, etc.), or intracardiac (e.g. limitation of myocardial perfusion or of chronotropic response), [23]. The extra- and intracardiac causes can be combined, as e.g. in the chronically increased afterload, requiring the ventricle to develop a higher active stress, i.e. to work chronically on a higher inotropic (contractility) level [5]. As presented in Section 2.1 accessing the tissue contractility requests employing a biophysical model of heart. Quantifying the contractility at rest and during physical exercise (which can be replaced by a pharmacological stress test) allows to assess the so-called contractile reserve (i.e. the level to which the contractility can increase in a given patient).

In [24], Ruijsink et al. performed a quantification of pharmacological stress exams in a group of patients born with a single ventricle. Their ventricles were surgically adjusted to serve as a pump for systemic and pulmonary circulations connected in series, by redirecting the main systemic veins (superior and inferior venae cavae) directly into the pulmonary arteries (the so-called total cavo-pulmonary connection known as the Fontan circulation [25]). Exercise intolerance is a relatively common early symptom of the failure of the single ventricle, and conventional methods used in clinics such as echocardiography or magnetic resonance imaging (MRI) 


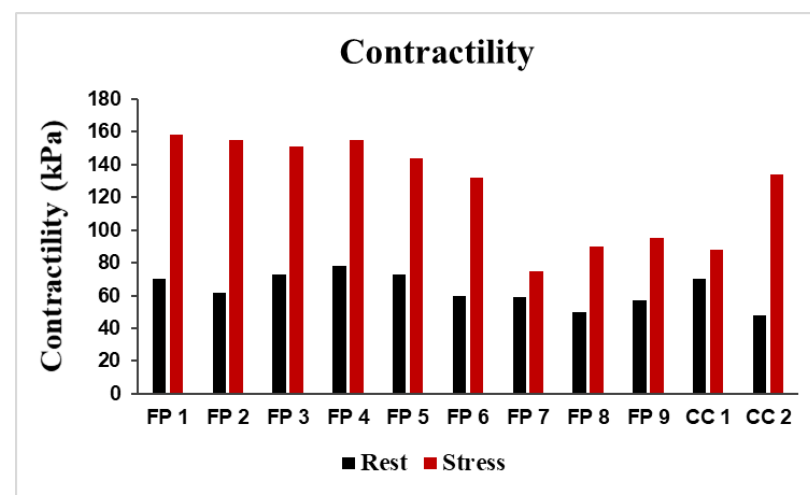

Fig. 4 Estimated contractilites at rest and under dobutamine test in 9 Fontan patients (FP) and 2 biventricular control cases (CC).

are often unable to define the actual causes of the onset of the failure. Simultaneous cardiac catheterization with MRI at rest and under pharmacological stress (simulating the exercise) has great potential to distinguish the actual cause of the intolerance. However, the complex physiology of the Fontan circulation, and large inter-individual variations makes it difficult to obtain direct conclusions from these investigations, even though having such rich datasets.

Employing a combined cardiac and circulatory model (similarly to Section 2.1p) allowed for an enhanced diagnostic assessment of pathophysiological exercise responses in patients with Fontan circulation. Fig. 4 reveals that the myocardial contractility values in Fontan patients were normal at rest. There was a good contractile reserve in most patients. The increase of contractility in three patients (marked as FP 7, 8, 9 and CC 1, we recall Fig. 4 was, however, limited. The low contractile reserve in these patients could be the cause of their early-stage HF. Good correlations of model-derived myocardial contractilites and periphery vascular resistance with their measured clinical surrogates depicted in Fig. 5 validate the modeling framework.

The work by Ruijsink et al. [24] demostrates that modeling can provide valuable additional information to the current diagnostic assessment of Fontan patients. This work paves the way for the translation of the model-accessed myocardial contractility and contractile reserve into other cohorts of patients, such as rTOF.

\subsection{Model-augmented monitoring during the perioperative period or in the ICU}

Real-time (or close to real-time) biomechanical models allow for coupling with monitoring data during general anesthesia (GA) or in the ICU. Direct analysis of the arterial pressure or cardiac output may be challenging in complex clinical situations. Patient-specific biomechanical modeling allows to simulate ventricular PV loops 

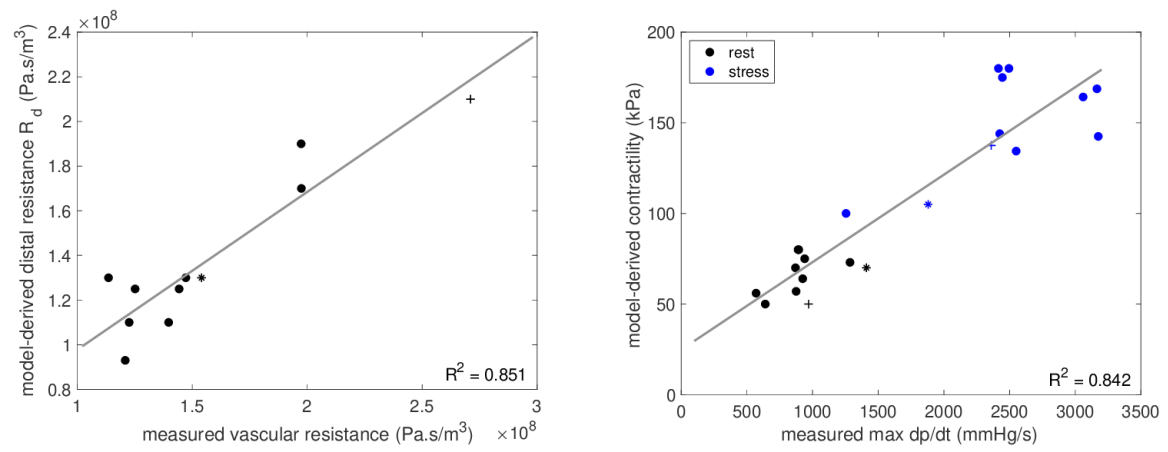

Fig. 5 Model estimated quantities vs measurements. Left: Model-derived distal Windkessel resistance $\left(R_{d}\right)$ versus measured vascular resistance at rest. Right: Model estimated contractility versus measured ventricular max dp/dt (i.e. the usual clinical surrogate of myocardial contractility). Figure used from [24] under the Creative Commons Attribution (CC BY) license.

and obtain functional indicators of the cardiovascular system, e.g. resistance of the circulatory system or myocardial contractility, evolving throughout GA or during the stay at ICU.

In [26], Le Gall et al. described a proof-of-concept study of applying biomechanical models to augment hemodynamic physiological monitoring. Patient-specific biomechanical models of heart and vasculature were created for 45 patients undergoing GA. While aortic pressure and flow were used to set up the patient-specific models, those then allowed for a quantitative assessment of heart function in each individual patient. The validation was conducted on a group of 4 patients, who underwent a combined MRI and pressure catheter procedure. Fig. 6(a) compares the PV loop generated by the model with the measured ventricular pressure and volume and demonstrates validation of the approach. Fig. 6(b) shows the model-derived PV loop of a patient under GA during hypotension and how the PV loop changed after administering the vasoactive drug norepinephrine and Fig. 7 shows the quantitative changes of physiology accessed thanks to modeling.

Work [26] by Le Gall et al. demonstrates the application of biophysical models built for individual patients to estimate PV loops and cardiovascular functional indicators using data, which are available during GA. Moreover, the predictive capability of the biophysical models representing the cardiovascular system of individual patients builds the foundations for a pro-active use in optimal clinical handling of complex situations (e.g. assistance in clinical management of septic shock).

Both increasing the insight in pathophysiology and proposing a possible therapeutic intervention could be helpful in newly discovered diseases. For instance, there is currently a limited knowledge about Multisystem Inflammatory Syndrome in Children (MIS-C) known also as Pediatric Inflammatory Multisystem Syndrometemporally associated with SARS-CoV-2 (PIMS-TS). It is a serious condition, which is associated to the SARS-Cov-2 virus and appearing typically 2-4 weeks after the exposure to the coronavirus, while the COVID-19 disease may pass only with mild 


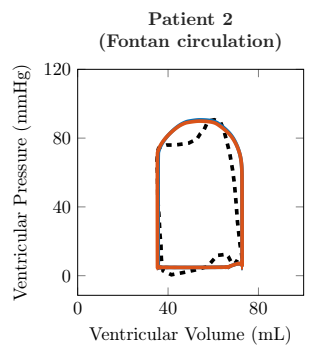

(a) Validation PV loops

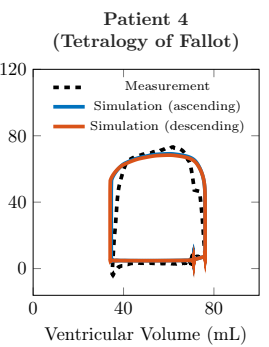

(b) PV loop at hypotension vs. after administering vasoactive drug norepinephrine

Fig. 6 Cardiac modeling coupled with monitoring signals. Figure used from [26] under the Creative Commons Attribution (CC BY) license.

symptoms [27, 28]. Model-augmented cardiovascular monitoring could be of a direct benefit in this life-threatening condition during the acute stage at ICU and also during the long-term clinical follow up of patients. Both were demonstrated in a study by Waugh et al. [29], see Fig. 8 . The patient-specific models of 8 patients allowed to access the cardiovascular physiology of MIS-C patients at ICU to distinguish between the cardiac and vascular component of their cardiovascular shock state. Secondly, the study demonstrated cardiac recovery 2-4 weeks after discharge from ICU, supporting the optimistic scenario that the heart is fully recovering within a month.

\subsection{Assessment of a long-term cardiac performance}

An assessment of a long-term cardiac performance has been studied within a kinematic growth theory framework as originally proposed by Rodriguez et al. [30], and later employed in the heart by Kroon et al. [31], and others [32, 33, 34, 4]. The growth and remodeling of the cardiac tissue is modeled as alterations in shape and size of the ventricular chamber in response to stress and / or strain-based stimuli. The model hypothesizes that concentric growth (thickening of the myocardial wall) occurs due to the stress-based stimuli (e.g. pressure overload) and eccentric growth due to the strain-based stimuli (volume overload). A number of studies have applied kinematic growth laws on the finite element anatomical models of ventricles to study the chronic changes of ventricular morphology in response to simulated aortic stenosis and mitral valve regurgitation (Kerckhoffs et al. [34]), or in response to simulated diastolic and systolic heart failure (Genet et al. [4]). In both studies model-predicted chronic changes in wall thickness, chamber size and geometry were in agreement with experimental data. Lee et al. [35] used the kinematic growth concept to study the reversible growth (reverse remodeling) in human left ventricle. 

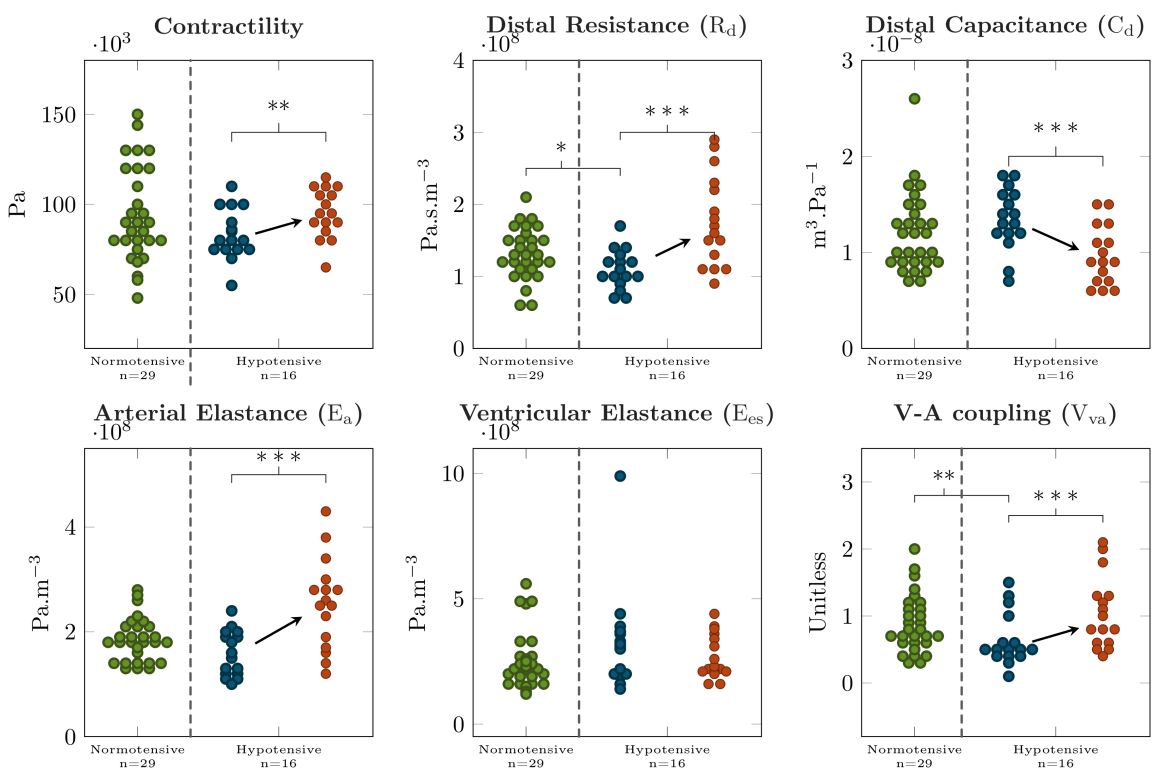

$\underset{.10^{8}}{\text { Ventricular Elastance }\left(\mathrm{E}_{\mathrm{es}}\right)}$

V-A coupling $\left(\mathrm{V}_{\mathrm{va}}\right)$
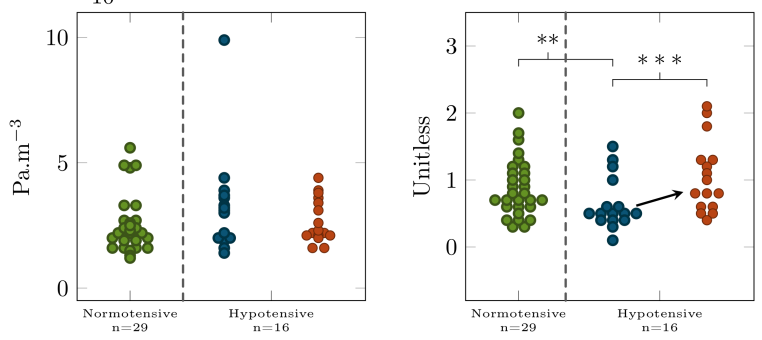

Internal Work $\left(I_{\mathrm{w}}\right)$

External Work ( $\left.\mathrm{E}_{\mathrm{w}}\right)$

Cardiac Efficiency (CE)
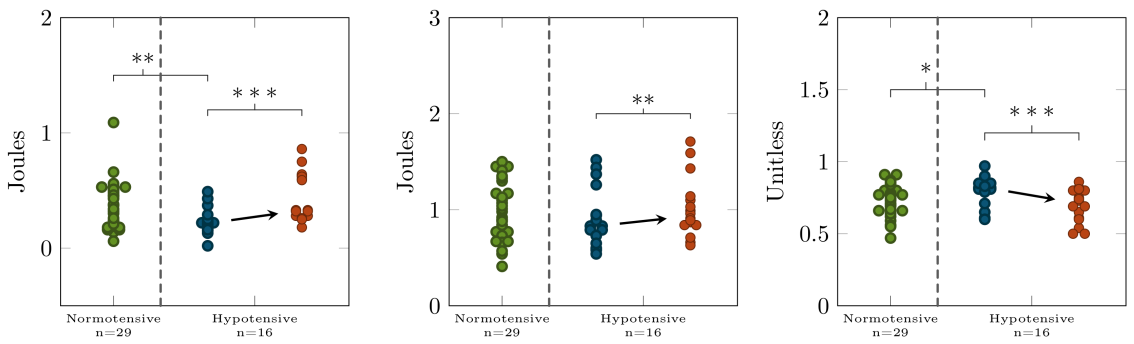

Fig. 7 Boxplots of model parameters and the results of the simulation, normotensive patients are in green; hypotensive patients before and after administering norepinephrine are in blue and red color, respectively. $* p<0.05$; ** $p<0.01 ; * * * p<0.001$. Figure used from [26] under the Creative Commons Attribution (CC BY) license.

The simulated response of end-diastolic pressure-volume relationship to unloading qualitatively reproduced experimental and clinical observations. To date kinematic growth theory is capable of predicting the consequence of growth on organ morphology while is limited in predicting tissue-level mechanical properties (e.g. myocardial contractility or stiffness) and micro-structural changes (e.g. apposition of collagen fibers). Hybrid modeling approaches linking tissue-level turnover events with an evolving organ morphology could be a way to better understand the mechanics of growth and remodeling in physiological and pathological conditions. For example, microstructurally oriented models such as constrained mixture theory originally designed to capture mass turnover events in individual constituents of the mixture (e.g. solid tissue) [36, 37], if applied to the heart could potentially describe e.g. 


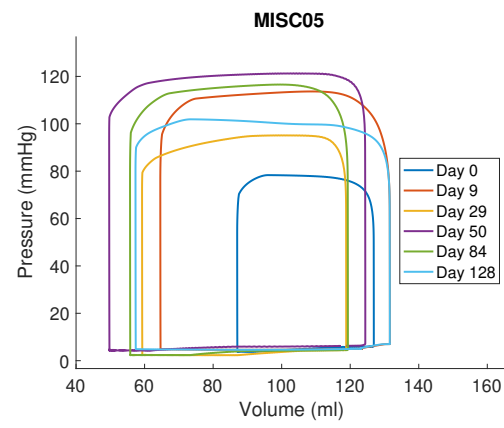

(a) PV loops

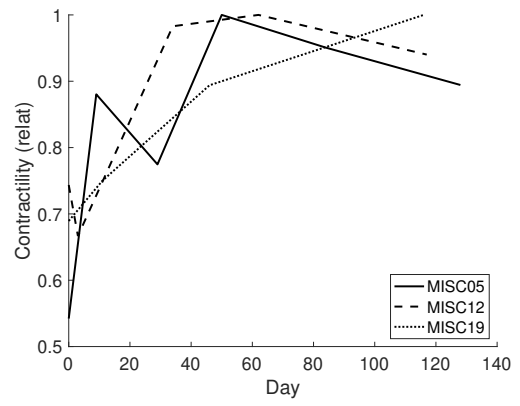

(b) Long-term evolution of myocardial contractility

Fig. 8 (a) Example of left ventricular PV loops in a selected patient with multisystem inflammatory syndrome in children (MIS-C) at acute stage and during the recovery. (b) Progressive recovery of myocardial contractility after MIS-C.

deposition/apposition of myofilaments or collagen in hypertensive ventricles. In the field of vascular modeling a number of attempts to combine a constrained mixture theory with kinematic growth have shown promising results in modeling the effect of elastin and collagen degradation on the development of thick-walled aortic aneurysms [38, 39]. Patient-specific modeling approach presented in Sections 2.1. 2.3 could evaluate current-state tissue-level mechanical properties and if combined with kinematic growth framework (or with a hybrid of kinematic growth and mixture theory) it has a potential to become an invaluable therapeutic tool in predicting long-term effects of therapies, e.g. response to PVR, and in predicting the optimal timing for interventions, including for complex surgeries.

\section{Pulmonary right ventricular resynchronization in congenital heart disease}

RV dysfunction and occasional failure are associated with a number of congenital heart lesions, most predominantly but not limited to rTOF. They are attributed to several factors including myocardial fibrosis due to preoperative hypoxemia and pressure overload, surgical scar and long-term post-repair volume overload caused by pulmonary regurgitation and frequently associated with RBBB [40]. RBBB is also the most frequent cause of electromechanical dyssynchrony in congenital heart disease. In rTOF patients, RV electromechanical dyssynchrony resembles the classic dyssynchrony pattern incorporating an early septal contraction with RV free wall pre-stretch followed by late free wall contraction and septal rebound stretch. It has been associated with a decreased RV ejection fraction, mechanical inefficiency and impaired exercise capacity [41, 42]. The dyssynchrony is hypothesized to play a 
significant role in the progress of RV dysfunction. A recently published study using computer modeling showed that the extent of negative influence of RV dyssynchrony may be greater than previously thought and may even overweight the pulmonary regurgitation during exercise [43]. A relief of RV volume overload by PVR is thought to reverse the pathological RV remodeling occurring due to the pulmonary regurgitation in rTOF patients. However, a decreased probability of reverse remodeling has been reported in patients with high RV end-diastolic and end-systolic volumes, low RV ejection fraction and those with a wide QRS complex ( $\geq 160 \mathrm{~ms}$ ) [44]. Thus, PVR alone may not lead to the normalization of RV performance.

Cardiac resynchronization therapy (CRT) has so far been used to treat dyssynchrony in the failing systemic (left) ventricle. Robust clinical data demonstrate a favorable CRT effect on acute hemodynamics, reverse cardiac remodeling, functional capacity and patient survival. Limited evidence suggests a similar CRT effect in children and patients with congenital heart disease [45]. Clinical reports on subpulmonary RV resynchronization (RV-CRT) are scarce, however. In this section we briefly review current experience with RV-CRT in patients with congenital heart disease.

\subsection{Temporary RV-CRT}

Published data show that acute RV-CRT through either RV or biventricular pacing improves short-term hemodynamics both in children and adults with congenital heart disease and RBBB. RV-CRT has been successfully used to treat RV dysfunction in acute postoperative setting [46]. Pacing is applied by temporary epicardial leads placed by a surgeon on the right atrium and the late activated free wall of the right ventricle. RV resynchronization is achieved by atrial-triggered RV free wall pacing in complete fusion with spontaneous ventricular activation to obtain a maximal QRS duration shortening. Specific attention is paid to a complete abolition of the broad S wave in standard lead I. The aim is to resynchronize electrically as well as mechanically the septum and RV free wall. A commercially available external dual-chamber pacemaker may be used. Temporary RV-CRT performed in this way carried a significant short-term improvement of hemodynamics in children early after surgery for TOF and may be a useful non-pharmacologic adjunct to the management of hemodynamically compromised patients. The resynchronization effect was maximized when pacing from the area of the latest RV activation. In another study, the temporary RV-CRT brought multiple positive effects on RV mechanics, synchrony and contraction efficiency [47]. 


\subsection{Permanent RV-CRT}

The use of permanent RV-CRT to treat RV dysfunction associated with RBBB seems to be the next logical step. Unfortunately, published evidence is limited. In the first well documented report on permanent RV-CRT in a patient late after the repair of TOF [48] both right and left ventricular function improved significantly within 6 months accompanied by an improvement in the NYHA class as well as exercise capacity. Data on a small series of congenital heart disease patients undergoing RVCRT has been presented at the HeartRhythm conference in 2018 [49] confirming multiple positive mid-term resynchronization effects on the RV systolic function, electromechanical synchrony and contraction efficiency.

\subsection{Towards electromechanical modeling for RV-CRT}

Cardiac modeling was employed in several projects predicting the effect of CRT on the failing LV [50, 51]. Pilot modeling study [52] demonstrates that pacing has potential to significantly decrease valvular regurgitation also in other pathologies - e.g. severe tricuspid regurgitation. Similar models are likely to be used in rTOF patients [43].

RV-CRT seems to be a valuable method to treat both acute and chronic subpulmonary right ventricular dysfunction and failure associated with RBBB in patients with congenital heart disease. Further studies are needed to better define its role in long-term RV failure management. Cardiac electromechanical models represent a great potential in optimization of this treatment.

\section{Model-constrained image processing}

One of the key image processing tasks in the field of medical imaging is image registration. It is defined as the process of aligning two or more images of the same scene. It is used to deal with misalignment caused by different time of acquisition, different scaling or different acquisition techniques. Aligning series of images taken at different times is often the first step in any subsequent analysis. However, the analysis of the detected misalignment itself might bear useful information. This applies specifically in cardiac applications, where both aspects of image registration are utilized. Firstly, aligning a given series of the cardiac images allows for estimation of local tissue specification that is encoded in the image series. Secondly, it is used to extract the motion of the moving organ from the image series. Features of interest, such as time evolution of volume and torsion, are obtained by the subsequent analysis of the detected motion.

Most image registration methods consist of defining a measure of misalignment and its minimization. The registration methods can be divided into several groups 
based on what is taken into account in the evaluation of misalignment [53]. These groups are: landmark-based methods; segmentation-based methods; and voxel-based methods. In the landmark-based registration methods the alignment of two images is evaluated based on the alignment of a finite set of voxels, the so-called landmarks, detected on both images. The landmarks can be detected automatically or manually, as in [54], where a landmark-based registration method is used for brain MR images. In the segmentation-based methods the alignment of images is evaluated based on the alignment of objects segmented in both images. In [55], B-spline method was used to register segmented left ventricle contours. Finally, in the voxel-based methods, the alignment is evaluated based on all voxels of the images. An optical flow method is a representative of this category. In [56], the optical flow method incorporating the image intensity constancy and gradient orientation was used for registration of multimodal images.

Mathematically speaking, image registration is an ill-posed problem and is therefore often formulated as a variational problem which typically contains a data sim-

ilarity term and a regularization term. The appropriate choice of the data similarity term is especially important when registering images obtained by different modalities or if the voxel intensity evolves in time. In such cases, the similarity cannot be evaluated based on the image intensity and more complex similarity terms are needed. Similarity terms based on correlation [57] or terms utilizing mutual information [58] are often employed. The regularization term deals with the ill-posedness of the problem. Moreover, it can be used to prevent non-physiological motion of the object. In advanced methods, the regularization term can incorporate physiological and physical knowledge of the processed object in the form of "model a priori knowledge".

In this section, we show some examples of both aspects of image registration: the extraction of motion indicators and estimation of the tissue characteristics indicators. In Sec. 4.1. we discuss the data similarity term used for registration of MOLLI MRI data (Modified Look-Locker inversion recovery, [59]), which are used to estimate the tissue $\mathrm{T} 1$ relaxation time (tissue characterization obtained by magnetic resonance related e.g. to assess myocardial fibrosis). In Sec. 4.2 we show the incorporation of a mechanical model into the regularization term. Another approach of constructing the data similarity term using directly a model of imaging technique is discussed in Sec. 4.3 The final parts represent crucial steps in patient-specific modeling: In Sec. 4.4 we briefly describe the problem of the fusion of clinical data and a complex biophysical model and in Sec. 4.5 we show some directions of employing machine learning and artificial intelligence.

\subsection{Assessment of T1 relaxation time from MOLLI MRI sequence}

In this section we show the approach proposed in [60] for the registration of MOLLI MR image series. The MOLLI sequence is used for pixel-wise estimation of the tissue characteristics of T1 relaxation time. In the case of suboptimal breath-holding, image 
registration is needed in order to compute reliable T1 relaxation times. This holds especially for the points adjacent to the epicardial and endocardial surfaces of the myocardium, where the misalignment may significantly corrupt the computation of the $\mathrm{T} 1$ relaxation time.

The evolution of image intensity and large changes in image contrast are the factors that make the registration of the image series acquired by MOLLI challenging. In order to deal with this characteristics of the sequence, a special data similarity term was proposed by Škardová et al. in [60], based on evaluating signed-distance of image voxels from segmented surfaces. The method requires the segmentation of epicardial and endocardial surface of the myocardium. The data similarity term is then based on the assumption

$$
\phi(\mathbf{x}(t), t)=\text { const } \quad \forall t \in(0, T),
$$

where $\phi(\mathbf{x}(t), t)$ represents the signed-distance of point $\mathbf{x}$ from the segmented contours of the myocardium. I.e., the distance of each point from the myocardium contour was assumed to remain the same over time. This formulation allows for the use of the voxel-based registration methods and excludes the effects of non-constant image intensity. Since the displacement caused by suboptimal breath-holding is independent of the frame ordering, all source images can be registered with the target image independently. Three images from the MOLLI series with segmented myocardium are shown in Fig. 9. The source images $S_{1}$ and $S_{2}$ are shown in Figs. 9(a-b), the target image $T$ in Fig. 9 (c). The result of registration using this method is depicted in Fig. 10.

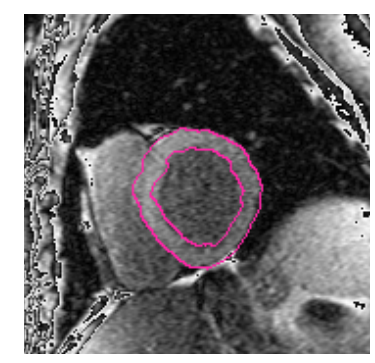

(a) Source image $S_{1}$

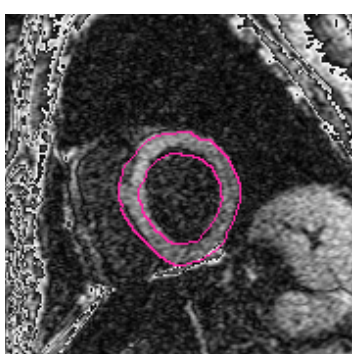

(b) Source image $S_{2}$

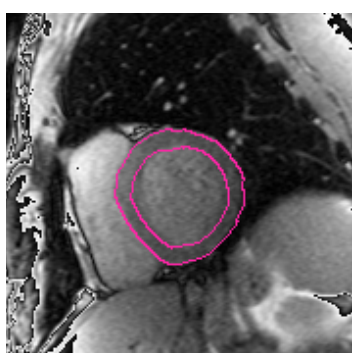

(c) Target image $T$

Fig. 9 Segmented epicardial and endocardial contours of myocardium of left ventricle. 


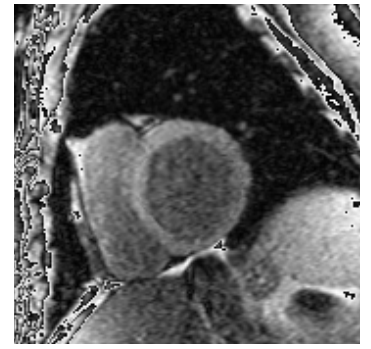

(a) Transformed source image (b) Transformed source image $S_{1}$

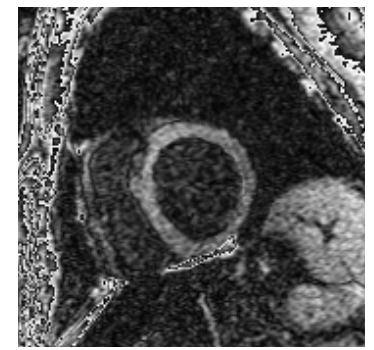

$S_{2}$

Fig. 10 Source images of Fig. 9 a-b) registered to target image Fig. 96. $)$.

\subsection{Motion extraction from image data using mechanical model constraints}

A detailed extraction of motion pattern from functional cine cardiovascular MR images (see Fig. 11) has been a challenging problem since longtime. In MRI, a special magnetic-saturation pulse can be applied. The tissue can be saturated in a regular pattern (typically in the form of lines or grids), with which the material points of the myocardium can be followed. The technique is known as SPAMM (SPAtial Modulation of Magnetization [61]) or as tagged MRI. The periodic character of the tag lines allows to use the so-called Harmonic Phase (HARP) [62] or Sine-Wave Modeling (SinMod) [63] analyses. Recently, an improved Harmonic Phase analysis method was introduced by Mella et al. under the name HARP-I [64]. HARP-I has a superior efficiency to the standard HARP or SinMod methods, as was demonstrated in [64]. However, it relies on acquiring the tagged MR images, which is practically difficult to do in every patient due to lengthening the MR exam.

Genet et al. [65] described a novel motion tracking based on image registration, while imposing mechanical constraint of equilibrated gap regularization - the socalled equilibrated warping 1 It has been shown to successfully track the motion not only in the tagged MR images but also to extract global torsion in standard cine MR images [66]. The LV torsion in rTOF patients was previously studied, however, poor reproducibility was observed when using standard clinical commercial software [67]. In [68], Castellanos et al. successfully employed the equilibrated warping in rTOF patients. In this work, the authors aimed to demonstrate that assessing the LV torsion in a robust and reproducible way has the potential to contribute to an earlier detection of heart failure.

\footnotetext{
${ }^{1}$ The implementation is available from: https://gitlab.inria.fr/mgenet/dolfin_dic
} 


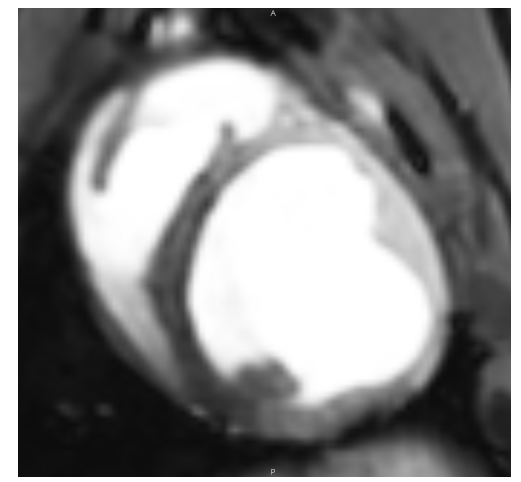

(a) Cine MRI: end-diastolic image

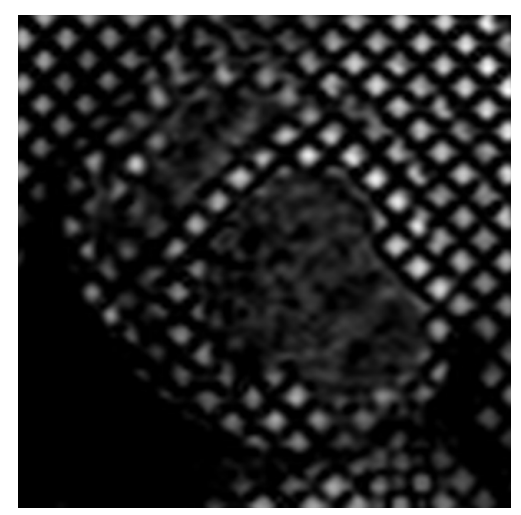

(c) Tagged MRI: end-diastolic image

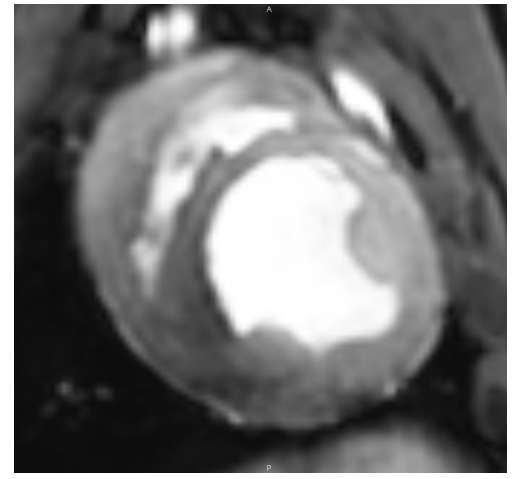

(b) Cine MRI: end-systolic image

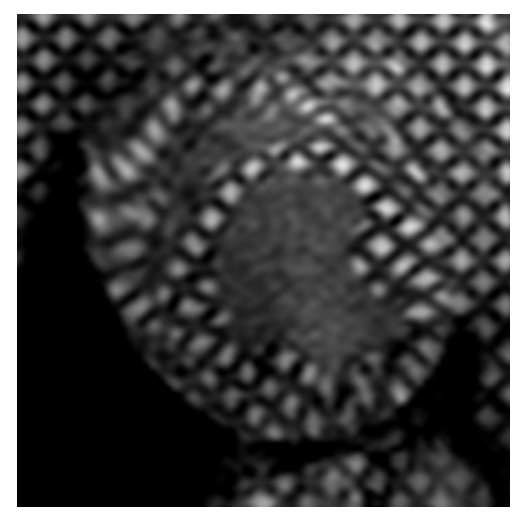

(d) Tagged MRI: end-systolic image

Fig. 11 Short axis views of cine MRI and tagged MRI.

\subsection{Motion extraction from image data using model of imaging modality}

In this section we discuss extracting motion from medical image series by using a model of the imaging modality. In [69], such an approach is employed to register 2D tagged MR images. This method uses a geometrical model of the segmented object that is to be registered and also the imaging model. For each setting of the geometrical model, the imaging model can be used to generate synthetic images. We look for the mapping that best matches the synthetic and acquired images.

The imaging model used by Škardová et al. in [69] includes the 2D tagging and artifacts caused by a limited image spatial resolution. The image registration is regularized by the equilibrated warping, as described in [65, 66]. We remark that the proposed method could be applied also for other medical imaging modalities. 
More effects could be considered, including complex artifacts given by the imaging method, e.g. if a very high acceleration of data acquisition is used. An efficient processing of such data has the potential to reduce the overall scanning time. This could substantially assist in some challenging MR acquisition such as cardiovascular MRI exam during physiological exercise [70].

\subsection{Model-data fusion}

Advanced image processing constrained by a model gives an opportunity for a fast and reliable extraction of motion from image data. This is important in stratifying patients (e.g. as presented in Sec.4.2). Moreover, the acquired and processed data can be incorporated into truly biophysical models in the sense of the third component of TCM (as listed in the introductory Section 1P - data-model fusion. The topic of assimilating clinical data into a complex biophysical model was reviewed by Chapelle et al. in [71]. The correction of model by data leads to increasing the patient-specificity of the models, as presented e.g. in [72, 73]. The biophysical character then allows to estimate some clinically relevant parameters contributing to a detailed description of physiology state. The parameters obtained thanks to fusing a model with data are represented for instance by passive tissue stiffness [74, 75, 76] or contractility [77, 78, 79] for the heart tissue. For large vessels, Young's modulus or relative pressures were estimated in [80, 81, 82], to give some examples. Such model-accessed parameters are expected to be included in clinical studies of a larger scale in the near future.

\subsection{Data-driven strategies for image analysis}

Recently, with an increasing availability of large amounts of medical image data, data-driven approaches have become more frequently used in multiple classes of medical image analysis. As examples can serve pattern recognition, segmentation and registration. The data-driven methods learn patterns directly from image data, therefore in principle, no mathematical model is needed. In practice, data-driven methods can be used alone or in combination with a mathematical model, if a model with an appropriate level of detail and expressiveness is available.

However, with the purely data-driven methods, problems with robustness and interpretability (which are key qualities in many medical applications) might arise. This could occur mainly for deep learning methods - neural networks with tens or even over hundreds of layers. Both approaches - model-based and data-driven - are compared in [83] in terms of robustness, accuracy, interpretability and explainability.

As was mentioned in the beginning of Section 4 the model-based image registration methods usually incorporate a data-similarity term and regularization terms. Recently, data-driven methods for learning transformation parameters by minimiz- 
ing traditional cost functions using neural networks have been presented. These methods can be divided into two groups: supervised - for which a set of registered images with known transformation parameters is available - and unsupervised - for which transformation parameters are learned directly form input image data. The supervised methods include for example a Spatial Pyramid Network (SPyNet) [84], a convolutional neural network (CNN) based method for optical flow estimation. Similarly to traditional optical flow method, SPyNet incorporates spatial resolution pyramid to bypass estimating large displacements. In [85] CNN based method for multimodal registration of $\mathrm{T} 1$ and $\mathrm{T} 2$-weighted MR images of brain was presented. The unsupervised methods based on CNN include [86], used for registration of brain MR images or [87] used for cardiac MR images.

Similarly, in image segmentation the data-driven methods learn the information about the shape and intensity distribution of a segmented object from the image data. Even early approaches using simple voxel-wise intensity features, for example the support vector machines (SVM) based method [88] and k-nearest neighbor classifier [89], were successfully used for brain segmentation. In classical machine learning approaches, the most effective methods are based on decision forests [90], which allow to incorporate larger feature vectors, as they perform feature selection and learning at the same time. Today, most of the successful segmentation methods are based on neural networks, specifically CNNs [91].

\section{Large-vessel flow modeling}

Phase-contrast magnetic resonance imaging (PC-MRI) [92] is used to assess the level of valvular stenosis or regurgitation [93]. PC-MRI is based on the fact that the phase shift of the MR signal after applying a bipolar gradient is linearly proportional to the velocity of the moving spins in the image. The linearity of the phase shift is valid only under the assumption of laminar flow and the measured flow is underestimated if the level of turbulence is above a certain level [94]. There exist research MR sequences specifically designed for turbulent flow (see e.g. [95, 96, 97]). However, they are currently not a part of standard clinical MR packages and cannot be therefore routinely used. Furthermore, they would substantially lengthen the MR exam, which would complicate their clinical deployment.

To reduce the limitations of standard PC-MRI, the imaging method can be coupled with a biophysical model of flow, in the sense of Section 4.4 The model can lead to a correction of data, e.g. of the underestimation of velocity in turbulent flow mentioned above. While some parts of the vessel geometry may be inaccessible to imaging, due to e.g. the presence of vascular stents as illustrated in Fig. 12, the computational flow dynamics (CFD) model constrained by the data measured before and after the stent has the potential to contribute to detecting the possible re-stenosis of the stented vessel segment. 


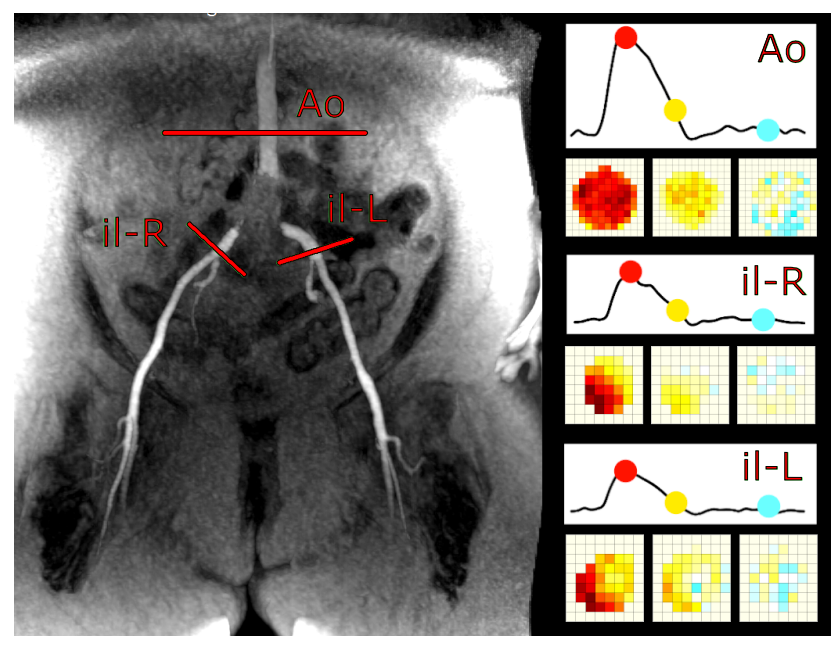

Fig. 12 Illustration of clinical PC-MRI examination of the bifurcation of abdominal aorta. Due to the presence of stents, the aortic bifurcation cannot be directly assessed. A CFD simulation has the potential to provide crucial information missing in the clinical assessment. Ao: Aorta; il-R: right common illiac artery; il-L: left common illiac artery.

\subsection{Phantom experiment}

Prior to clinical usage of a numerical model of flow in vessels, it should be validated on representative problems using in vivo or in vitro data [98]. In vivo data often suffer from beat-to-beat flow changes, limited signal-to-noise ratio and motion artifacts [99]. In vitro experiments, however, can be controlled in several ways. They provide valuable information firstly for the validation of a CFD model and secondly for a successful coupling of the model with PC-MRI data. The CFD and experimental data can be compared using common statistical methods applied on quantities of interest, e.g. mean flow perpendicular to a centerline at various locations along the vessel, or flow ratio between two or more branches of the vessel tree [100]. Prior to comparison, experimental data may be used to fine-tune the CFD model parameters, such as fluid density and viscosity [100]. It can also facilitate setting a boundary condition, e.g. whether a simple mean flow is sufficient as an input and output condition or whether a more elaborate flow profile is needed. Or, similarly, whether a zero outlet pressure provides sufficient results as opposed to a more precise estimate, such as $1 \mathrm{D}$ in silico pressure model [100, 101]. In the following paragraphs, we provide some practical aspects of in vitro experimenting.

Firstly, a flow rate close to the physiological regime should be achieved. A special care must be taken to utilize a stable pump that does not experience a decrease in power when subjected to an increasing resistance. The sources of resistance are hydrostatic pressure (caused by the vertical arrangement of the pump and MRI table); and the resistance of the circulatory system (causing a pressure loss). To minimize the friction of fluid in the circulation, a hose of a large diameter and a minimum 
possible length should be used. This is limited by the amount of fluid available and the necessity of placing the pump outside the magnetic field of the MR scanner. Any connecting parts of the system or devices, such as a flow meter, also tend to add a resistance. Inevitable internal friction in the fluid itself is another factor influencing the pump performance.

PC-MR images may suffer from low velocity-to-noise ratio. This statistical error of velocity can be minimized by prolonged scan times. Such an approach would be often non-feasible in vivo. Not only precision but also the accuracy is a challenge of in vitro measurement especially with smaller vessels as they are prone to partial volume effect. Matching the magnitude signal of vessel wall and of the fluid ameliorates this artifact [102].

Another advantage of in vitro experiments is the precise knowledge of geometry. Phantoms can be created from a 3D printed model using suitable silicon-based materials [103]. The geometry of the model can then be described analytically or obtained by using a more precise imaging modality, such as computed tomography.

Initial validation phases of a flow model can be conducted by using simplified in vitro experiments. For instance, it may be sufficient to consider constant flow through a rigid pipe with a valve-shaped orifice (narrowing), while using clean water as a medium. Such example is illustrated in Fig.13 and was used in the study by Fučík et al. [104]. The shapes of phantom valves represent three degrees of valvular stenosis (mild, moderate and severe). Hessenthaler et al. presented in [105] a phantom with a non-rigid walls suitable for assessing the computational models including fluidstructure interaction. Finally, various numerical benchmarks (e.g. [106]) can be used to evaluate the implementation issues of models.

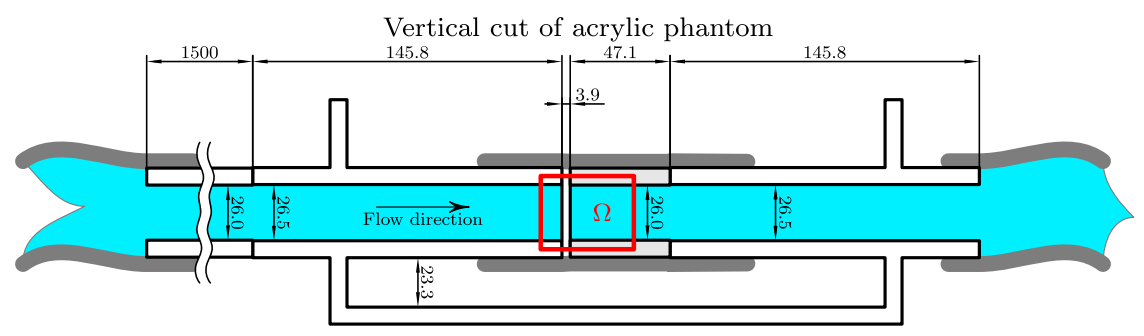

severe stenosis

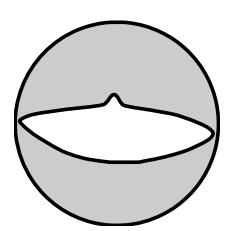

moderate stenosis

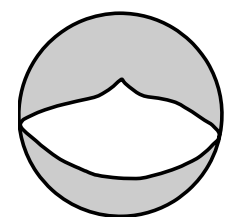

mild stenosis

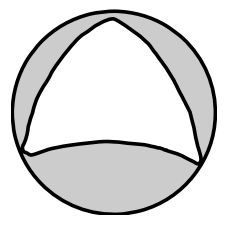

Fig. 13 Illustration of vertical cross section through experimental phantom and acrylic plates mimicking the mild (Mild-VS), moderate (Mod-VS) and severe (Sev-VS) stenoses. All dimension are in $\mathrm{mm}$. The red region $\Omega$ represents the domain for the computational simulations in 3D. 
For validation of more complex flow models, the initial experiment can be further modified. For instance, a pulsating flow pump can be used or the water medium can be replaced by some blood-like fluid. There exist commercially available bloodmimicking fluids suitable for the use in MRI. Their price may, however, be an obstacle. Several substitutes were proposed. In [107] Campo-Deaño et al. explore the properties of xantham gum to mimic the shear-thinning behavior and of glycerin and succrose to mimic the elasticity. These alternatives are cheap and easy to prepare. However, they are less convenient to work with as they are difficult to rinse off in the case of leak and they also deteriorate with time.

\subsection{Computational fluid dynamic model}

Applications of CFD in cardiovascular medicine can be found e.g. in the treatment and diagnosis of aortic or cerebral aneurysm [108], aortic coarctation [109], vessel or valvular stenosis [110, 111], valvular regurgitation [112], or a description of the aortic arch flow [113]. The CFD is usually coupled with PC-MRI [114], where the measurement serves as the source of initial and boundary conditions for the CFD. Classical CFD methods (such as the finite volume or finite element methods) are widely used methods and were applied in the cardiovascular problems using, for example, commercial ANSYS engineering software [115] or open-source software OpenFOAM [116]. The main disadvantage of these classical CFD methods is their substantial computational cost (for reasonable spatial and temporal resolutions) which is a key obstacle for their application during medical examinations.

The lattice Boltzmann method (LBM) [117] can be considered as a modern alternative to the classical numerical methods. The main advantage of LBM is the accuracy and local nature which allows the massive parallel implementation on computational clusters, including those with modern graphic accelerator units (GPUs), and clusters of GPUs. LBM has been already used in a wide range of computational problems. In [118], Fučík et al. showed the capability of LBM to simulate fluid flow in complex fluid domains such as aortic vessels with stenosis.

In [104], Fučík et al. address the issue of underestimating the backflow by PCMRI. The 3D geometry was given by the geometry of the phantom with three levels of stenosis, see Fig. 13. The inlet boundary condition was given by the flow measured prior to the stenosed region. By comparing the PC-MRI measured backflow with the simulation, excellent agreement between LBM and PC-MRI results of both forward and backward flow for mild and moderate stenosis was observed, as depicted in Fig 14. However, a large discrepancy occurred in the case of the severe stenosis, as can be seen in Fig. 15. This observation can be explained by the decrease in signal caused by the turbulence-induced accumulation of spins with different encoded phases in one voxel during the readout period. The presented work is an essential initial step towards combining measured data by PC-MRI with CFD model suitable for the use in clinical workflow, allowing to enhance the interpretation of measured data by a biophysical model. 

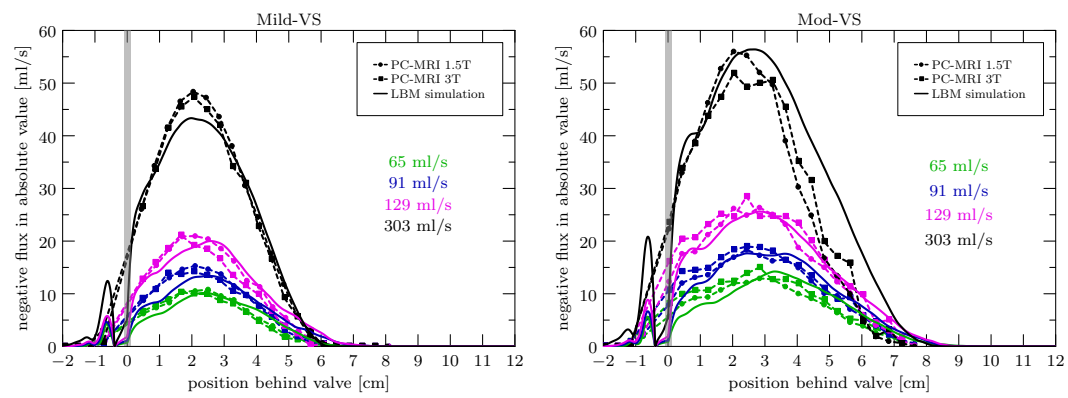

Fig. 14 Simulated backward flow for mild (left) and moderate stenosis (1.5T and 3T MRI systems) confronted with the simulation by lattice Boltzmann method. The solid vertical line represents the valve position. Each color corresponds to the flow rate on the inlet given by the measurement ranging from physiological at rest (green) up to accelerated flow (black) representing e.g. high exercise stress.

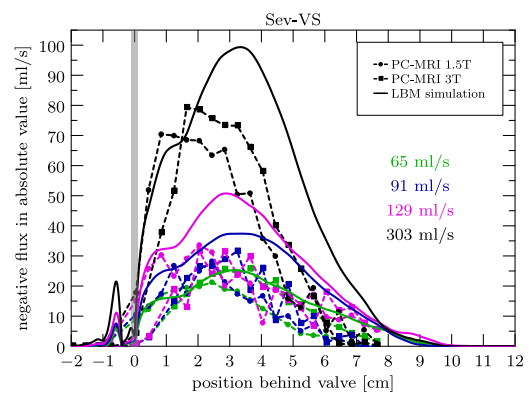

Fig. 15 Simulated backward flow for severe stenosis (1.5T and 3T MRI systems) confronted with the simulation by lattice Boltzmann method. The solid vertical line represents the valve position. Each color corresponds to the flow rate on the inlet given by the measurement ranging from physiological at rest (green) up to accelerated flow (black) representing e.g. high exercise stress.

The appropriate CFD model should reflect the correct physical properties of the fluid in the vessels. Thus, Škardová et al. investigated in [119] three fluid models for the blood flow through a stenotic vessel. Two models approximated blood as a Newtonian fluid with different fluid kinematic viscosity. The third one used the Carreau-Yasuda model [120] which is designed for capturing the non-Newtonian behavior of blood.

The simulations based on the non-Newtonian model in three different aorta geometries with gradually increasing severities of stenosis showed that the severity of stenosis has a non-negligible impact on fluid viscosity. Nevertheless, the fluctuation of viscosity were small in all simulations. Thus, based on the numerical simulations, the Newtonian approach can be applied to the fluid simulations in the geometries of large vessels, however, with a careful choice of the Newtonian fluid viscosity. Phantom experiments (e.g. as described in Section 5.1) with various types of fluid 
will be the next step to shed light into the most appropriate constitutive model of blood in vessels.

\section{Discussion}

This chapter primarily dealt with TCM applied in congenital heart diseases. It focused on tetralogy of Fallot after complete surgical repair (rTOF). TOF may be a relatively rare disease, however, the regular follow up exams of patients represent a significant cost for the healthcare system. Furthermore, the acquired data throughout the evolution of cardiovascular remodeling provide an opportunity to address some important clinical questions of TOF using cardiovascular models.

Sections 2 5 addressed some common issues in rTOF patients. Each topic is typically advanced by one or a few research groups. However, in a given individual patient, the optimal management may require several of these approaches (as well as some additional ones) to be put together - one of the challenges of successful clinical application of cardiovascular modeling nowadays.

While TOF represents one particular disease, a number of already existing modeling techniques can be applied to the cohort of TOF. On the other hand, the novel techniques developed specifically in the group of TOF patients could be useful for other pathologies as well. Indeed, each challenge solved in the TOF cohort has its counterparts in a much larger group of acquired heart diseases. This is in line with the TOFMOD acronym - Tetralogy of Fallot and Modeling of Diseases - as is depicted in the schematics in Fig. 16 For instance:

1. Knowledge obtained from the optimization of PVR could possibly be translated into a much larger cohort of the left-heart valve (aortic and mitral) pathologies [121].

2. Optimal timing of intervention on pulmonary artery could contribute in optimizing management of pathologies affecting the aorta, head and neck aortic branches or e.g. in the interventions for ischemic disease of lower extremities.

3. Biomechanical modeling has been shown to be feasible in the planning and optimization of cardiac resynchronization therapy (CRT) for failing LV in acquired heart disease [50, 51]. This can be a starting point for the translation of CRT modeling into the TOF cohort, for whom the therapy is focused on the right ventricle (RV-CRT) [47].

4. Thanks to regular follow up of rTOF patients in cardiology clinic, the failing LV or RV might be diagnosed and treated in its early stage. The diagnosis and management of an early-stage HF could then serve also for the cohort of the acquired heart diseases, in order to shift the management of their heart failure into earlier stages. This has the potential to improve the current 5-year survival rate of HF being around $45 \%$ [122].

This chapter demonstrated the potential of the biomechanical modeling approaches to assist in data handling and inform about a decease progress. To ensure 


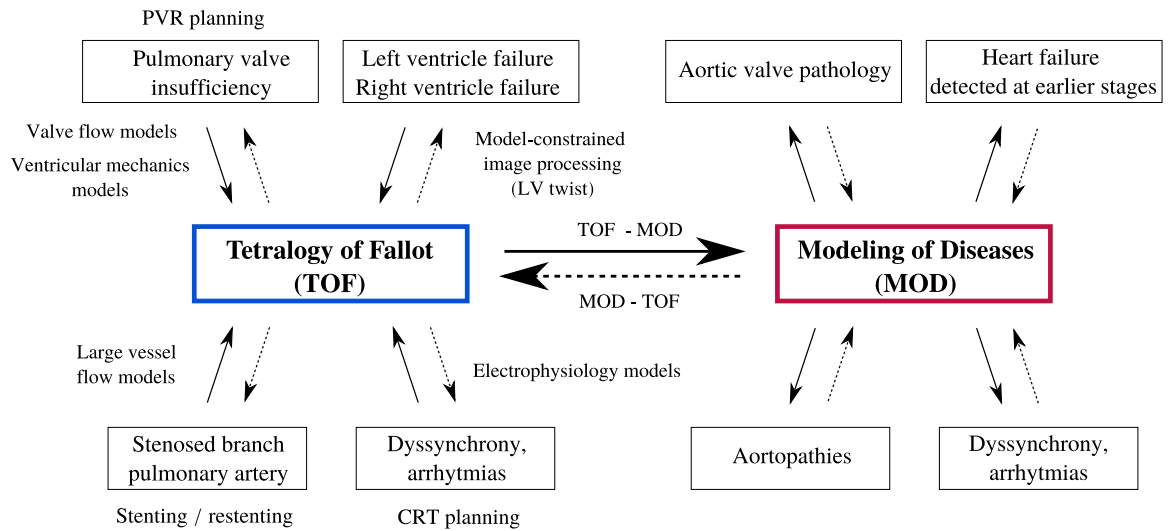

Fig. 16 Tetralogy of Fallot and Modeling of Diseases (TOFMOD) scheme.

reliable predictions the rigorous assessment of the credibility of the model must be included in the workflow. Namely, the quantification of the model ability to explain the underlying mechanism must be performed in terms of verification of the numerical method, validation of the biophysical assumptions of the model (a degree to which the model is capable to represent the reality, usually performed with a measured dataset), and uncertainty quantification [123] - a subject of the recent research within the cardiac modeling community. When personalizing the models (the so-called data-model coupling) the clinical data serving as input for the model are subject to the measurement errors. Therefore, in order to correctly interpret the model-derived predictions it is of paramount importance to understand and quantify the sensitivity of the model outputs to the noisy input data signals. For example, the study of Eck et al. [124] demonstrated the efficiency of stochastic mathematical solutions, namely the polynomial chaos method (for details see [125]), to quantify the uncertainty of the parameters of one-dimensional patient-specific blood flow model.

The uttermost goal of precision medicine is to minimize uncertainties in the patient management workflow. The creation of "digital twins" or "avatars" of the patients [126] and their subsequent holistic translation into clinical practice could be optimized via synergistic approach of combining physics-based and statistical models. These two approaches could counterbalance limitations present in either of them. For example, a physics-based model can be used when a solid understanding of the biophysical system and the validation data are available (e.g. prediction of immediate adaptation of tissue contractility in response to PVR [17]). However, should the underlying biophysical mechanism be poorly understood (e.g long-term tissue remodeling) or being too complex to be described by the model a statistical approach may still be capable of identifying some predictive relations (e.g. statistical shape modeling to predict RV remodeling [127] or characterizing RV-LV interactions [128] in rTOF patients). The synergistic approach was used in recent work by Regazzoni et al. [129] to explain an unknown long-term dynamics of the aortic flow 
in hypertensive conditions. A mechanical model of large-vessel flow (capturing the short-term phenomena) was combined with data-driven modeling approach (machine learning) to explain the long-term phenomena.

\section{Conclusion}

Applying modeling methods in clinic is a very recent research topic with a number of associated challenges. First, a relevant clinical question, which can be addressed by modeling, must be defined. Second, the model including suitable components and of an adequate complexity must be selected. Third, data have to be obtainable, allowing for model personalization. When turning a model into the patient-specific setup, the physical and physiological character of the models allows them to estimate quantities that are hard (or even impossible) to measure in vivo, while maintaining clinical relevance. Secondly, the predictive capabilities of such models allow to assess the action of certain therapeutic effects in silico. Both have the potential to contribute into optimal clinical management of patients. Clinical problems requesting to employ novel techniques typically represent rather large challenges. Combining various multi-disciplinary approaches in a collaborative way has the potential to advance the clinical management and can be helpful for patients.

Acknowledgements The authors acknowledge the support of the Associated Team TOFMOD, created between Inria France and UT Southwestern Medical Center Dallas; the Ministry of Health of the Czech Republic (project No. NV19-08-00071); the Ministry of Education, Youth and Sports of the Czech Republic (under the OP RDE grant number CZ.02.1.01/0.0/0.0/16_019/0000765:Research Center for Informatics); and the Grant Agency of the Czech Technical University in Prague (grant No. SGS20/184/OHK4/3T/14).

The authors would like to thank their co-workers in the research projects described in this chapter, in particular to Dominique Chapelle, Martin Genet, Philippe Moireau (research team MEDISIM, Inria, France); Arthur Le Gall, Etienne Gayat, Fabrice Vallée (Dept. of Anesthesia and Critical Care, Lariboisière hospital Paris, France); Camille Hancock Friesen, Joshua Greer, Gerald Greil, Animesh Tandon, Rebecca Waugh (Dept. of Pediatrics, UT Southwestern Medical Center, Dallas, TX); Daniel Castellanos (Children's Boston Hospital, MA); Kuberan Pushparajah, Bram Ruijsink, Konrad Zugaj (St Thomas' Hospital, King's College London, UK); Jakub Klinkovský, Petr Pauš (Faculty of Nuclear Sciences and Physical Engineering, Czech Technical University in Prague, Czech Republic) and Robert Straka (AGH University of Science and Technology, Krakow, Poland). In addition, the authors would like to thank to Federica Caforio (Gottfried Schatz Research Center, Division of Biophysics, Medical University of Graz, Graz, Austria) for her valuable comments.

\section{References}

1. R. Chabiniok, V. Y. Wang, M. Hadjicharalambous, L. Asner, J. Lee, M. Sermesant, E. Kuhl, A. A. Young, P. Moireau, M. P. Nash, et al., "Multiphysics and multiscale modelling, datamodel fusion and integration of organ physiology in the clinic: ventricular cardiac mechanics," Interface focus, vol. 6, no. 2, p. 20150083, 2016. 
2. R. H. Anderson, S. Webb, N. A. Brown, W. Lamers, and A. Moorman, "Development of the heart:(3) formation of the ventricular outflow tracts, arterial valves, and intrapericardial arterial trunks," Heart, vol. 89, no. 9, pp. 1110-1118, 2003.

3. E. Fallot, "Contribution a l'anatomie pathologique de la maladie bleue (cyanotic cardiaque)," Marseille méd, vol. 25, pp. 77-138, 1888.

4. M. Genet, L. C. Lee, B. Baillargeon, J. M. Guccione, and E. Kuhl, "Modeling pathologies of diastolic and systolic heart failure," Annals of biomedical engineering, vol. 44, no. 1, pp. 112-127, 2016.

5. J. E. Hall and M. E. Hall, Guyton and Hall textbook of medical physiology e-Book. Elsevier Health Sciences, 2020.

6. L. Johnson, M. A. Simon, M. R. Pinsky, and S. G. Shroff, "Insights into the effects of contraction dyssynchrony on global left ventricular mechano-energetic function," Pacing and Clinical Electrophysiology, vol. 32, no. 2, pp. 224-233, 2009.

7. G. D. Nollert, S. H. Däbritz, M. Schmoeckel, C. Vicol, and B. Reichart, "Risk factors for sudden death after repair of tetralogy of Fallot," The Annals of thoracic surgery, vol. 76, no. 6, pp. 1901-1905, 2003.

8. T. Oosterhof, F. J. Meijboom, H. W. Vliegen, M. G. Hazekamp, A. H. Zwinderman, B. J. Bouma, A. P. van Dijk, and B. J. Mulder, "Long-term follow-up of homograft function after pulmonary valve replacement in patients with tetralogy of Fallot," European heart journal, vol. 27, no. 12, pp. 1478-1484, 2006.

9. M. M. Cheung, I. E. Konstantinov, and A. N. Redington, "Late complications of repair of tetralogy of Fallot and indications for pulmonary valve replacement," in Seminars in thoracic and cardiovascular surgery, vol. 17, pp. 155-159, Elsevier, 2005.

10. M. A. Gatzoulis, J. A. Till, J. Somerville, and A. N. Redington, "Mechanoelectrical interaction in tetralogy of Fallot: QRS prolongation relates to right ventricular size and predicts malignant ventricular arrhythmias and sudden death," Circulation, vol. 92, no. 2, pp. 231-237, 1995.

11. A. Sabate Rotes, H. M. Connolly, C. A. Warnes, N. M. Ammash, S. D. Phillips, J. A. Dearani, H. V. Schaff, H. M. Burkhart, D. O. Hodge, S. J. Asirvatham, et al., "Ventricular arrhythmia risk stratification in patients with tetralogy of Fallot at the time of pulmonary valve replacement," Circulation: Arrhythmia and Electrophysiology, vol. 8, no. 1, pp. 110$116,2015$.

12. J. P. Bokma, T. Geva, L. A. Sleeper, S. V. B. Narayan, R. Wald, K. Hickey, K. Jansen, R. Wassall, M. Lu, M. A. Gatzoulis, et al., "A propensity score-adjusted analysis of clinical outcomes after pulmonary valve replacement in tetralogy of Fallot," Heart, vol. 104, no. 9, pp. 738-744, 2018.

13. T. Geva, B. Mulder, K. Gauvreau, S. V. Babu-Narayan, R. M. Wald, K. Hickey, A. J. Powell, M. A. Gatzoulis, and A. M. Valente, "Preoperative predictors of death and sustained ventricular tachycardia after pulmonary valve replacement in patients with repaired tetralogy of Fallot enrolled in the INDICATOR cohort," Circulation, vol. 138, no. 19, pp. 2106-2115, 2018.

14. E. A. Nielsen, M. Sun, O. Honjo, V. E. Hjortdal, A. N. Redington, and M. K. Friedberg, "Dual Endothelin Receptor Blockade Abrogates Right Ventricular Remodeling and Biventricular Fibrosis in Isolated Elevated Right Ventricular Afterload," PLOS ONE, vol. 11, no. 1, p. e0146767, 2016

15. G. Nollert, T. Fischlein, S. Bouterwek, C. Böhmer, W. Klinner, and B. Reichart, "Long-term survival in patients with repair of tetralogy of Fallot: 36-year follow-up of 490 survivors of the first year after surgical repair," Journal of the American College of Cardiology, vol. 30, no. 5, pp. 1374-1383, 1997.

16. V. Wang, P. Nielsen, and M. Nash, "Image-based predictive modeling of heart mechanics," Annual review of biomedical engineering, vol. 17, pp. 351-383, 2015.

17. M. Gusseva, T. Hussain, C. Hancock Friesen, P. Moireau, A. Tandon, G. Greil, K. Hasbani, D. Chapelle, and R. Chabiniok, "Biomechanical modeling to inform pulmonary valve replacement in tetralogy of Fallot patients after complete repair," Canadian Journal of Cardiology, 2021. 
18. M. Caruel, R. Chabiniok, P. Moireau, Y. Lecarpentier, and D. Chapelle, "Dimensional reductions of a cardiac model for effective validation and calibration," Biomechanics and Modeling in Mechanobiology, vol. 13, pp. 897-914, Aug. 2014.

19. N. Stergiopulos, B. E. Westerhof, and N. Westerhof, "Total arterial inertance as the fourth element of the windkessel model," American Journal of Physiology-Heart and Circulatory Physiology, vol. 276, pp. H81-H88, Jan. 1999.

20. J. Sainte-Marie, D. Chapelle, R. Cimrman, and M. Sorine, "Modeling and estimation of the cardiac electromechanical activity," Computers \& Structures, vol. 84, pp. 1743-1759, Nov. 2006.

21. D. Chapelle, P. Le Tallec, P. Moireau, and M. Sorine, "An energy-preserving muscle tissue model: formulation and compatible discretizations," International Journal for Multiscale Computational Engineering, vol. 10, no. 2, pp. 189-211, 2012.

22. P. Lurz, J. Nordmeyer, V. Muthurangu, S. Khambadkone, G. Derrick, R. Yates, M. Sury, P. Bonhoeffer, and A. M. Taylor, "Comparison of Bare Metal Stenting and Percutaneous Pulmonary Valve Implantation for Treatment of Right Ventricular Outflow Tract Obstruction," Circulation, vol. 119, pp. 2995-3001, June 2009.

23. A. T. Yetman, K.-J. Lee, R. Hamilton, W. R. Morrow, and B. W. McCrindle, "Exercise capacity after repair of tetralogy of Fallot in infancy," American Journal of Cardiology, vol. 87, no. 8, pp. 1021-1023, 2001.

24. B. Ruijsink, K. Zugaj, J. Wong, K. Pushparajah, T. Hussain, P. Moireau, R. Razavi, D. Chapelle, and R. Chabiniok, "Dobutamine stress testing in patients with Fontan circulation augmented by biomechanical modeling," PLoS one, vol. 15, no. 2, p. e0229015, 2020.

25. P. Khairy, N. Poirier, and L.-A. Mercier, "Univentricular heart," Circulation, vol. 115, no. 6, pp. 800-812, 2007.

26. A. Le Gall, F. Vallée, K. Pushparajah, T. Hussain, A. Mebazaa, D. Chapelle, E. Gayat, and R. Chabiniok, "Monitoring of cardiovascular physiology augmented by a patient-specific biomechanical model during general anesthesia. A proof of concept study," PLoS one, vol. 15, no. 5, p. e0232830, 2020.

27. M. Ahmed, S. Advani, A. Moreira, S. Zoretic, J. Martinez, K. Chorath, S. Acosta, R. Naqvi, F. Burmeister-Morton, F. Burmeister, et al., "Multisystem inflammatory syndrome in children: A systematic review," EClinicalMedicine, vol. 26, p. 100527, 2020.

28. Z. Belhadjer, M. Méot, F. Bajolle, D. Khraiche, A. Legendre, S. Abakka, J. Auriau, M. Grimaud, M. Oualha, M. Beghetti, et al., "Acute heart failure in multisystem inflammatory syndrome in children (MIS-C) in the context of global SARS-CoV-2 pandemic," Circulation, 2020.

29. R. Waugh, M. Abdelghafar Hussein, J. Weller, K. Sharma, G. Greil, J. Kahn, T. Hussain, and R. Chabiniok, "Cardiac modeling for Multisystem Inflammatory Syndrome in Children (MIS-C, PIMS-TS)," in International Conference on Functional Imaging and Modeling of the Heart (FIMH), pp. 435-446, Springer, 2021.

30. E. K. Rodriguez, A. Hoger, and A. D. McCulloch, "Stress-dependent finite growth in soft elastic tissues.," Journal of biomechanics, vol. 27, pp. 455-467, Apr. 1994.

31. W. Kroon, T. Delhaas, T. Arts, and P. Bovendeerd, "Computational modeling of volumetric soft tissue growth: application to the cardiac left ventricle," Biomechanics and Modeling in Mechanobiology, vol. 8, pp. 301-309, Aug. 2009.

32. S. Göktepe, O. J. Abilez, and E. Kuhl, "A generic approach towards finite growth with examples of athlete's heart, cardiac dilation, and cardiac wall thickening," Journal of the mechanics and physics of solids, vol. 58, pp. 1661-1680, Oct. 2010.

33. S. Göktepe, O. J. Abilez, K. K. Parker, and E. Kuhl, "A multiscale model for eccentric and concentric cardiac growth through sarcomerogenesis," Journal of Theoretical Biology, vol. 265, no. 3, pp. 433-442, 2010.

34. R. C. P. Kerckhoffs, J. Omens, and A. D. McCulloch, "A single strain-based growth law predicts concentric and eccentric cardiac growth during pressure and volume overload.," Mechanics research communications, vol. 42, pp. 40-50, June 2012. 
35. L. C. Lee, M. Genet, G. Acevedo-Bolton, K. Ordovas, J. M. Guccione, and E. Kuhl, "A computational model that predicts reverse growth in response to mechanical unloading," Biomechanics and Modeling in Mechanobiology, vol. 14, no. 2, pp. 217-229, 2015.

36. J. A. Niestrawska, C. M. Augustin, and G. Plank, "Computational modeling of cardiac growth and remodeling in pressure overloaded hearts - Linking microstructure to organ phenotype," Acta biomaterialia, vol. 106, pp. 34-53, 2020.

37. G. A. Ateshian and J. D. Humphrey, "Continuum mixture models of biological growth and remodeling: past successes and future opportunities," Annual review of biomedical engineering, vol. 14, pp. 97-111, 2012.

38. P. W. Alford, J. D. Humphrey, and L. A. Taber, "Growth and remodeling in a thick-walled artery model: effects of spatial variations in wall constituents," Biomechanics and Modeling in Mechanobiology, vol. 7, pp. 245-262, Aug. 2008.

39. W. J. Lin, M. D. Iafrati, R. A. Peattie, and L. Dorfmann, "Growth and remodeling with application to abdominal aortic aneurysms," Journal of Engineering Mathematics, vol. 109, pp. 113-137, Apr. 2018.

40. Task Force on the Management of Grown Up Congenital Heart Disease of the European Society of Cardiology, J. Deanfield, E. Thaulow, C. Warnes, G. Webb, F. Kolbel, A. Hoffman, K. Sorenson, H. Kaemmerer, U. Thilen, et al., "Management of grown up congenital heart disease," European heart journal, vol. 24, no. 11, pp. 1035-1084, 2003.

41. W. Hui, C. Slorach, A. Dragulescu, L. Mertens, B. Bijnens, and M. K. Friedberg, "Mechanisms of right ventricular electromechanical dyssynchrony and mechanical inefficiency in children after repair of tetralogy of Fallot," Circulation: Cardiovascular Imaging, vol. 7, no. 4, pp. 610618, 2014.

42. D. Yim, W. Hui, G. Larios, A. Dragulescu, L. Grosse-Wortmann, B. Bijnens, L. Mertens, and M. K. Friedberg, "Quantification of right ventricular electromechanical dyssynchrony in relation to right ventricular function and clinical outcomes in children with repaired tetralogy of Fallot," Journal of the American Society of Echocardiography, vol. 31, no. 7, pp. 822-830, 2018.

43. J. Lumens, C.-P. S. Fan, J. Walmsley, D. Yim, C. Manlhiot, A. Dragulescu, L. GrosseWortmann, L. Mertens, F. W. Prinzen, T. Delhaas, and M. K. Friedberg, "Relative impact of right ventricular electromechanical dyssynchrony versus pulmonary regurgitation on right ventricular dysfunction and exercise intolerance in patients after repair of tetralogy of Fallot," Journal of the American Heart Association, vol. 8, no. 2, p. e010903, 2019.

44. T. Geva, K. Gauvreau, A. J. Powell, F. Cecchin, J. Rhodes, J. Geva, and P. del Nido, "Randomized trial of pulmonary valve replacement with and without right ventricular remodeling surgery," Circulation, vol. 122, no. 11_suppl_1, pp. S201-S208, 2010.

45. K. S. Motonaga and A. M. Dubin, "Cardiac resynchronization therapy for pediatric patients with heart failure and congenital heart disease: a reappraisal of results," Circulation, vol. 129, no. 18, pp. 1879-1891, 2014.

46. P. Vojtovič, F. Kučera, P. Kubuš, R. Gebauer, T. Matějka, T. Tláskal, M. Ložek, J. Kovanda, and J. Janoušek, "Acute right ventricular resynchronization improves haemodynamics in children after surgical repair of tetralogy of Fallot," Ep Europace, vol. 20, no. 2, pp. 323-328, 2018.

47. J. Janoušek, J. Kovanda, M. Ložek, V. Tomek, P. Vojtovič, R. Gebauer, P. Kubuš, M. Krejččŕ, J. Lumens, T. Delhaas, et al., "Pulmonary right ventricular resynchronization in congenital heart disease: acute improvement in right ventricular mechanics and contraction efficiency," Circulation: Cardiovascular Imaging, vol. 10, no. 9, p. e006424, 2017.

48. P. Kubuš, O. Materna, P. Tax, V. Tomek, and J. Janoušek, "Successful permanent resynchronization for failing right ventricle after repair of tetralogy of Fallot," Circulation, vol. 130, no. 22, pp. e186-e190, 2014.

49. J. Janoušek, J. Kovanda, M. Ložek, V. Tomek, R. Gebauer, and P. Kubuš, "Cardiac resynchronization therapy for treatment of chronic pulmonary right ventricular dysfunction in congenital heart disease," in HeartRhythm, vol. 15(suppl), p. S270, 2018. Abstract.

50. S. A. Niederer, G. Plank, P. Chinchapatnam, M. Ginks, P. Lamata, K. S. Rhode, C. A. Rinaldi, R. Razavi, and N. P. Smith, "Length-dependent tension in the failing heart and the efficacy 
of cardiac resynchronization therapy," Cardiovascular research, vol. 89, no. 2, pp. 336-343, 2010.

51. M. Sermesant, R. Chabiniok, P. Chinchapatnam, T. Mansi, F. Billet, P. Moireau, J.-M. Peyrat, K. Wong, J. Relan, K. Rhode, et al., "Patient-specific electromechanical models of the heart for the prediction of pacing acute effects in CRT: a preliminary clinical validation," Medical image analysis, vol. 16, no. 1, pp. 201-215, 2012.

52. R. Chabiniok, P. Moireau, C. Kiesewetter, T. Hussain, R. Razavi, and D. Chapelle, "Assessment of atrioventricular valve regurgitation using biomechanical cardiac modeling," in International Conference on Functional Imaging and Modeling of the Heart (FIMH), pp. 401-411, Springer, 2017.

53. J. A. Maintz and M. A. Viergever, "A survey of medical image registration," Medical image analysis, vol. 2, no. 1, pp. 1-36, 1998.

54. C. Hogea, C. Davatzikos, and G. Biros, "Brain-tumor interaction biophysical models for medical image registration," SIAM Journal on Scientific Computing, vol. 30, no. 6, pp. 30503072, 2008.

55. X. Deng and T. S. Denney, "Three-dimensional myocardial strain reconstruction from tagged MRI using a cylindrical B-spline model," IEEE Transactions on Medical Imaging, vol. 23, no. 7, pp. 861-867, 2004.

56. M. Heinrich, J. Schnabel, F. Gleeson, F. Brady, and M. Jenkinson, "Non-rigid multimodal medical image registration using optical flow and gradient orientation," Proc. Medical Image Analysis and Understanding, pp. 141-145, 2010.

57. A. Roche, G. Malandain, X. Pennec, and N. Ayache, "The correlation ratio as a new similarity measure for multimodal image registration," in International Conference on Medical Image Computing and Computer-Assisted Intervention, pp. 1115-1124, Springer, 1998.

58. X. Lu, S. Zhang, H. Su, and Y. Chen, "Mutual information-based multimodal image registration using a novel joint histogram estimation," Computerized Medical Imaging and Graphics, vol. 32, no. 3, pp. 202-209, 2008.

59. D. R. Messroghli, A. Radjenovic, S. Kozerke, D. M. Higgins, M. U. Sivananthan, and J. P. Ridgway, "Modified Look-Locker inversion recovery (MOLLI) for high-resolution T1 mapping of the heart," Magnetic Resonance in Medicine, vol. 52, no. 1, pp. 141-146, 2004.

60. K. Škardová, T. Oberhuber, J. Tintěra, and R. Chabiniok, "Signed-distance function based non-rigid registration of image series with varying image intensity," Discrete \& Continuous Dynamical Systems - S, vol. 14, no. 3, pp. 1145-1160, 2021.

61. L. Axel and L. Dougherty, "MR imaging of motion with spatial modulation of magnetization," Radiology, vol. 171, no. 3, pp. 841-845, 1989.

62. N. F. Osman, W. S. Kerwin, E. R. McVeigh, and J. L. Prince, "Cardiac motion tracking using CINE harmonic phase (HARP) magnetic resonance imaging," Magnetic Resonance in Medicine, vol. 42, no. 6, pp. 1048-1060, 1999.

63. T. Arts, F. W. Prinzen, T. Delhaas, J. R. Milles, A. C. Rossi, and P. Clarysse, "Mapping displacement and deformation of the heart with local sine-wave modeling," IEEE transactions on medical imaging, vol. 29, no. 5, pp. 1114-1123, 2010.

64. H. Mella, J. Mura, H. Wang, M. D. Taylor, R. Chabiniok, J. Tintera, J. Sotelo, and S. Uribe, "HARP-I: A harmonic phase interpolation method for the estimation of motion from tagged MR images," IEEE transactions on medical imaging, vol. 40, no. 4, pp. 1240-1252, 2021.

65. M. Genet, C. T. Stoeck, C. Von Deuster, L. C. Lee, and S. Kozerke, "Equilibrated warping: finite element image registration with finite strain equilibrium gap regularization," Medical image analysis, vol. 50, pp. 1-22, 2018.

66. L. C. Lee and M. Genet, "Validation of equilibrated warping-image registration with mechanical regularization-on 3D ultrasound images," in International Conference on Functional Imaging and Modeling of the Heart (FIMH), pp. 334-341, Springer, 2019.

67. B. E. Burkhardt, M. N. Velasco Forte, S. Durairaj, I. Rafiq, I. Valverde, A. Tandon, J. Simpson, and T. Hussain, "Timely pulmonary valve replacement may allow preservation of left ventricular circumferential strain in patients with tetralogy of Fallot," Frontiers in Pediatrics, vol. 5 , p. 39, 2017. 
68. D. A. Castellanos, K. Škardová, A. Bhattaru, G. Greil, A. Tandon, J. Dillenbeck, B. Burkhardt, T. Hussain, M. Genet, and R. Chabiniok, "Left ventricular torsion obtained using equilibrated warping in patients with repaired tetralogy of Fallot," Pediatric Cardiology, 2021.

69. K. Škardová, M. Rambausek, R. Chabiniok, and M. Genet, "Mechanical and imaging modelsbased image registration," in ECCOMAS Thematic Conference on Computational Vision and Medical Image Processing, pp. 77-85, Springer, 2019.

70. B. Ruijsink, E. Puyol-Antón, M. Usman, J. van Amerom, P. Duong, M. N. V. Forte, K. Pushparajah, A. Frigiola, D. A. Nordsletten, A. P. King, et al., "Semi-automatic cardiac and respiratory gated MRI for cardiac assessment during exercise," in Molecular Imaging, Reconstruction and Analysis of Moving Body Organs, and Stroke Imaging and Treatment, pp. 86-95, Springer, 2017.

71. D. Chapelle, M. Fragu, V. Mallet, and P. Moireau, "Fundamental principles of data assimilation underlying the Verdandi library: applications to biophysical model personalization within euHeart," Medical \& biological engineering \& computing, vol. 51, no. 11, pp. 1221-1233, 2013.

72. R. Chabiniok, G. Bureau, A. Groth, J. Tintera, J. Weese, D. Chapelle, and P. Moireau, "Cardiac displacement tracking with data assimilation combining a biomechanical model and an automatic contour detection," in International Conference on Functional Imaging and Modeling of the Heart (FIMH), pp. 405-414, Springer, 2019.

73. P. Moireau, C. Bertoglio, N. Xiao, C. A. Figueroa, C. Taylor, D. Chapelle, and J.-F. Gerbeau, "Sequential identification of boundary support parameters in a fluid-structure vascular model using patient image data," Biomechanics and modeling in mechanobiology, vol. 12, no. 3, pp. 475-496, 2013.

74. V. Y. Wang, H. Lam, D. B. Ennis, B. R. Cowan, A. A. Young, and M. P. Nash, "Modelling passive diastolic mechanics with quantitative MRI of cardiac structure and function," Medical image analysis, vol. 13, no. 5, pp. 773-784, 2009.

75. M. Hadjicharalambous, R. Chabiniok, L. Asner, E. Sammut, J. Wong, G. Carr-White, J. Lee, R. Razavi, N. Smith, and D. Nordsletten, "Analysis of passive cardiac constitutive laws for parameter estimation using 3D tagged MRI," Biomechanics and modeling in mechanobiology, vol. 14, no. 4, pp. 807-828, 2015.

76. M. Hadjicharalambous, L. Asner, R. Chabiniok, E. Sammut, J. Wong, D. Peressutti, E. Kerfoot, A. King, J. Lee, R. Razavi, et al., "Non-invasive model-based assessment of passive left-ventricular myocardial stiffness in healthy subjects and in patients with non-ischemic dilated cardiomyopathy," Annals of biomedical engineering, vol. 45, no. 3, pp. 605-618, 2017.

77. R. Chabiniok, P. Moireau, P.-F. Lesault, A. Rahmouni, J.-F. Deux, and D. Chapelle, "Estimation of tissue contractility from cardiac cine-MRI using a biomechanical heart model," Biomechanics and modeling in mechanobiology, vol. 11, no. 5, pp. 609-630, 2012.

78. L. Asner, M. Hadjicharalambous, R. Chabiniok, D. Peresutti, E. Sammut, J. Wong, G. CarrWhite, P. Chowienczyk, J. Lee, A. King, et al., "Estimation of passive and active properties in the human heart using 3D tagged MRI," Biomechanics and modeling in mechanobiology, vol. 15, no. 5, pp. 1121-1139, 2016.

79. A. Imperiale, D. Chapelle, and P. Moireau, "Sequential data assimilation for mechanical systems with complex image data: application to tagged-MRI in cardiac mechanics," Advanced Modeling and Simulation in Engineering Sciences, vol. 8, no. 1, pp. 1-47, 2021.

80. C. Bertoglio, P. Moireau, and J.-F. Gerbeau, "Sequential parameter estimation for fluidstructure problems: Application to hemodynamics," International Journal for Numerical Methods in Biomedical Engineering, vol. 28, no. 4, pp. 434-455, 2012.

81. A. Caiazzo, F. Caforio, G. Montecinos, L. O. Muller, P. J. Blanco, and E. F. Toro, "Assessment of reduced-order unscented Kalman filter for parameter identification in 1-dimensional blood flow models using experimental data," International journal for numerical methods in biomedical engineering, vol. 33, no. 8, p. e2843, 2017.

82. F. Donati, S. Myerson, M. M. Bissell, N. P. Smith, S. Neubauer, M. J. Monaghan, D. A. Nordsletten, and P. Lamata, "Beyond Bernoulli: improving the accuracy and precision of 
noninvasive estimation of peak pressure drops," Circulation: Cardiovascular Imaging, vol. 10, no. 1, p. e005207, 2017.

83. D. Rueckert and J. A. Schnabel, "Model-based and data-driven strategies in medical image computing," Proceedings of the IEEE, vol. 108, no. 1, pp. 110-124, 2019.

84. A. Ranjan and M. J. Black, "Optical flow estimation using a spatial pyramid network," in Proceedings of the IEEE conference on computer vision and pattern recognition, pp. 41614170, 2017.

85. M. Simonovsky, B. Gutiérrez-Becker, D. Mateus, N. Navab, and N. Komodakis, "A deep metric for multimodal registration," in International conference on medical image computing and computer-assisted intervention, pp. 10-18, Springer, 2016.

86. G. Balakrishnan, A. Zhao, M. R. Sabuncu, J. Guttag, and A. V. Dalca, "An unsupervised learning model for deformable medical image registration," in Proceedings of the IEEE conference on computer vision and pattern recognition, pp. 9252-9260, 2018.

87. A. V. Dalca, G. Balakrishnan, J. Guttag, and M. R. Sabuncu, "Unsupervised learning for fast probabilistic diffeomorphic registration," in International Conference on Medical Image Computing and Computer-Assisted Intervention, pp. 729-738, Springer, 2018.

88. S. Bauer, L.-P. Nolte, and M. Reyes, "Fully automatic segmentation of brain tumor images using support vector machine classification in combination with hierarchical conditional random field regularization," in International conference on medical image computing and computer-assisted intervention (MICCAI), pp. 354-361, Springer, 2011.

89. H. A. Vrooman, C. A. Cocosco, F. van der Lijn, R. Stokking, M. A. Ikram, M. W. Vernooij, M. M. Breteler, and W. J. Niessen, "Multi-spectral brain tissue segmentation using automatically trained k-nearest-neighbor classification," Neuroimage, vol. 37, no. 1, pp. 71-81, 2007.

90. A. Montillo, J. Shotton, J. Winn, J. E. Iglesias, D. Metaxas, and A. Criminisi, "Entangled decision forests and their application for semantic segmentation of CT images," in Biennial International Conference on Information Processing in Medical Imaging, pp. 184-196, Springer, 2011.

91. W. Bai, M. Sinclair, G. Tarroni, O. Oktay, M. Rajchl, G. Vaillant, A. M. Lee, N. Aung, E. Lukaschuk, M. M. Sanghvi, et al., "Automated cardiovascular magnetic resonance image analysis with fully convolutional networks," Journal of Cardiovascular Magnetic Resonance, vol. 20 , no. 1 , p. $65,2018$.

92. K. S. Nayak, J.-F. Nielsen, M. A. Bernstein, M. Markl, P. D. Gatehouse, R. M. Botnar, D. Saloner, C. Lorenz, H. Wen, B. S. Hu, et al., "Cardiovascular magnetic resonance phase contrast imaging," Journal of Cardiovascular Magnetic Resonance, vol. 17, no. 1, p. 71, 2015.

93. P. Dyverfeldt, M. Bissell, A. J. Barker, A. F. Bolger, C.-J. Carlhäll, T. Ebbers, C. J. Francios, A. Frydrychowicz, J. Geiger, D. Giese, et al., "4D flow cardiovascular magnetic resonance consensus statement," Journal of Cardiovascular Magnetic Resonance, vol. 17, no. 1, p. 72, 2015.

94. K. R. O'Brien, B. R. Cowan, M. Jain, R. A. Stewart, A. J. Kerr, and A. A. Young, "MRI phase contrast velocity and flow errors in turbulent stenotic jets," Journal of Magnetic Resonance Imaging, vol. 28, no. 1, pp. 210-218, 2008.

95. A. J. Sederman, M. D. Mantle, C. Buckley, and L. F. Gladden, "MRI technique for measurement of velocity vectors, acceleration, and autocorrelation functions in turbulent flow," Journal of Magnetic Resonance, vol. 166, no. 2, pp. 182-189, 2004.

96. P. Dyverfeldt, R. Gårdhagen, A. Sigfridsson, M. Karlsson, and T. Ebbers, "On MRI turbulence quantification," Magnetic resonance imaging, vol. 27, no. 7, pp. 913-922, 2009.

97. J. Walheim, H. Dillinger, and S. Kozerke, "Multipoint 5D flow cardiovascular magnetic resonance-accelerated cardiac-and respiratory-motion resolved mapping of mean and turbulent velocities," Journal of Cardiovascular Magnetic Resonance, vol. 21, no. 1, pp. 1-13, 2019.

98. L. Zhong, J.-M. Zhang, B. Su, R. S. Tan, J. C. Allen, and G. S. Kassab, "Application of patient-specific computational fluid dynamics in coronary and intra-cardiac flow simulations: Challenges and opportunities," Frontiers in Physiology, vol. 9, p. 742, 2018. 
99. J. Lotz, C. Meier, A. Leppert, and M. Galanski, "Cardiovascular flow measurement with phase-contrast MR imaging: Basic facts and implementation,” RadioGraphics, vol. 22, no. 3, pp. 651-671, 2002. PMID: 12006694.

100. A. Roldán-Alzate, S. García-Rodríguez, P. V. Anagnostopoulos, S. Srinivasan, O. Wieben, and C. J. François, "Hemodynamic study of TCPC using in vivo and in vitro 4D flow MRI and numerical simulation," Journal of Biomechanics, vol. 48, no. 7, pp. 1325-1330, 2015.

101. P. D. Morris, A. Narracott, H. von Tengg-Kobligk, D. A. Silva Soto, S. Hsiao, A. Lungu, P. Evans, N. W. Bressloff, P. V. Lawford, D. R. Hose, and J. P. Gunn, "Computational fluid dynamics modelling in cardiovascular medicine," Heart, vol. 102, no. 1, pp. 18-28, 2016.

102. R. L. Wolf, R. L. Ehman, S. J. Riederer, and P. J. Rossman, "Analysis of systematic and random error in MR volumetric flow measurements," Magnetic Resonance in Medicine, vol. 30, no. 1, pp. 82-91, 1993.

103. P. Cao, Y. Duhamel, G. Olympe, B. Ramond, and F. Langevin, "A new production method of elastic silicone carotid phantom based on MRI acquisition using rapid prototyping technique," in 2013 35th Annual International Conference of the IEEE Engineering in Medicine and Biology Society (EMBC), pp. 5331-5334, IEEE, 2013.

104. R. Fučík, R. Galabov, P. Pauš, P. Eichler, J. Klinkovský, R. Straka, J. Tintěra, and R. Chabiniok, "Investigation of phase-contrast magnetic resonance imaging underestimation of turbulent flow through the aortic valve phantom: Experimental and computational study using lattice Boltzmann method," Magnetic Resonance Materials in Physics, Biology and Medicine, vol. 33, no. 5, pp. 649-662, 2020.

105. A. Hessenthaler, N. Gaddum, O. Holub, R. Sinkus, O. Röhrle, and D. Nordsletten, "Experiment for validation of fluid-structure interaction models and algorithms," International journal for numerical methods in biomedical engineering, vol. 33, no. 9, p. e2848, 2017.

106. R. Chabiniok, J. Hron, A. Jarolímová, J. Málek, K. Rajagopal, K. Rajagopal, H. Švihlová, and K. Tůma, "A benchmark problem to evaluate implementational issues for three-dimensional flows of incompressible fluids subject to slip boundary conditions," Applications in Engineering Science, p. 100038, 2021.

107. L. Campo-Deaño, R. P. Dullens, D. G. Aarts, F. T. Pinho, and M. S. Oliveira, "Viscoelasticity of blood and viscoelastic blood analogues for use in polydymethylsiloxane in vitro models of the circulatory system," Biomicrofluidics, vol. 7, no. 3, p. 034102, 2013.

108. J. R. Anderson, O. Diaz, R. Klucznik, Y. J. Zhang, G. W. Britz, R. G. Grossman, N. Lv, Q. Huang, and C. Karmonik, "Validation of computational fluid dynamics methods with anatomically exact, 3D printed MRI phantoms and 4D pcMRI," in 2014 36th Annual International Conference of the IEEE Engineering in Medicine and Biology Society, pp. 6699-6701, IEEE, 2014.

109. L. Goubergrits, E. Riesenkampff, P. Yevtushenko, J. Schaller, U. Kertzscher, F. Berger, and T. Kuehne, "Is MRI-Based CFD Able to Improve Clinical Treatment of Coarctations of Aorta?," Ann Biomed Eng, vol. 43, no. 1, pp. 168-176, 2015.

110. H. Ha, J. Lantz, M. Ziegler, B. Casas, M. Karlsson, P. Dyverfeldt, and T. Ebbers, "Evaluation of aortic regurgitation with cardiac magnetic resonance imaging: a systematic review," Sci Rep, vol. 7, p. 46618, 2017.

111. J. Kweon, D. H. Yang, G. B. Kim, N. Kim, M. Paek, A. F. Stalder, A. Greiser, and Y.-H. Kim, "Four-dimensional flow MRI for evaluation of post-stenotic turbulent flow in a phantom: comparison with flowmeter and computational fluid dynamics," Eur Radiol, vol. 26, no. 10, pp. 3588-3597, 2016.

112. D. C. Wendell, M. M. Samyn, J. R. Cava, M. M. Krolikowski, and J. F. LaDisa, "The Impact of Cardiac Motion on Aortic Valve Flow Used in Computational Simulations of the Thoracic Aorta," J Biomech Eng, vol. 138, no. 9, p. 091001, 2016.

113. S. Miyazaki, K. Itatani, T. Furusawa, T. Nishino, M. Sugiyama, Y. Takehara, and S. Yasukochi, "Validation of numerical simulation methods in aortic arch using 4D Flow MRI," Heart Vessels, vol. 32, no. 8, pp. 1032-1044, 2017.

114. J. Sotelo, L. Dux-Santoy, A. Guala, J. Rodríguez-Palomares, A. Evangelista, C. Sing-Long, J. Urbina, J. Mura, D. E. Hurtado, and S. Uribe, “3D axial and circumferential wall shear 
stress from 4D flow MRI data using a finite element method and a laplacian approach," Magn Reson Med, vol. 79, no. 5, pp. 2816-2823, 2018.

115. D. Hose, P. Lawford, A. Narracott, J. Penrose, and I. Jones, "Fluid-solid interaction: benchmarking of an external coupling of ANSYS with CFX for cardiovascular applications," Journal of medical engineering \& technology, vol. 27, no. 1, pp. 23-31, 2003.

116. S. Pinto, E. Doutel, J. Campos, and J. Miranda, "Blood analog fluid flow in vessels with stenosis: Development of an OpenFOAM code to simulate pulsatile flow and elasticity of the fluid," APCBEE procedia, vol. 7, pp. 73-79, 2013.

117. T. Krüger, H. Kusumaatmaja, A. Kuzmin, O. Shardt, G. Silva, and E. M. Viggen, "The lattice Boltzmann method," Springer International Publishing, vol. 10, no. 978-3, pp. 4-15, 2017.

118. R. Fučík, P. Eichler, R. Straka, P. Pauš, J. Klinkovský, and T. Oberhuber, "On optimal node spacing for immersed boundary-lattice Boltzmann method in 2D and 3D," Computers \& Mathematics with Applications, vol. 77, no. 4, pp. 1144-1162, 2019.

119. K. Škardová, P. Eichler, T. Oberhuber, and R. Fučík, "Investigation of blood-like nonNewtonian fluid flow in stenotic arteries using the lattice Boltzmann method in 2D," in Proceedings of ALGORITMY, pp. 101-110, 2020.

120. J. Boyd, J. M. Buick, and S. Green, "Analysis of the Casson and Carreau-Yasuda nonNewtonian blood models in steady and oscillatory flows using the lattice Boltzmann method," Physics of Fluids, vol. 19, no. 9, p. 093103, 2007.

121. P. Faggiano, G. P. Aurigemma, C. Rusconi, and W. H. Gaasch, "Progression of valvular aortic stenosis in adults: literature review and clinical implications," American heart journal, vol. 132, no. 2, pp. 408-417, 1996.

122. C. J. Taylor, J. M. Ordóñez-Mena, A. K. Roalfe, S. Lay-Flurrie, N. R. Jones, T. Marshall, and F. R. Hobbs, "Trends in survival after a diagnosis of heart failure in the United Kingdom 2000-2017: population based cohort study," BMJ, vol. 364, 2019.

123. M. Viceconti, F. Pappalardo, B. Rodriguez, M. Horner, J. Bischoff, and F. Musuamba Tshinanu, "In silico trials: Verification, validation and uncertainty quantification of predictive models used in the regulatory evaluation of biomedical products," Methods, vol. 185, pp. 120127, 2021. Methods on simulation in biomedicine.

124. V. Eck, J. Sturdy, and L. Hellevik, "Effects of arterial wall models and measurement uncertainties on cardiovascular model predictions," Journal of Biomechanics, vol. 50, pp. 188-194, 2017. Biofluid mechanics of multitude pathways: From cellular to organ.

125. V. G. Eck, W. P. Donders, J. Sturdy, J. Feinberg, T. Delhaas, L. R. Hellevik, and W. Huberts, "A guide to uncertainty quantification and sensitivity analysis for cardiovascular applications.," International journal for numerical methods in biomedical engineering, vol. 32, Aug. 2016.

126. J. Corral-Acero, F. Margara, M. Marciniak, C. Rodero, F. Loncaric, Y. Feng, A. Gilbert, J. F. Fernandes, H. A. Bukhari, A. Wajdan, et al., "The 'Digital Twin' to enable the vision of precision cardiology," European Heart Journal, 2020.

127. T. Mansi, I. Voigt, B. Leonardi, X. Pennec, S. Durrleman, M. Sermesant, H. Delingette, A. M. Taylor, Y. Boudjemline, G. Pongiglione, et al., "A statistical model for quantification and prediction of cardiac remodelling: Application to tetralogy of Fallot," IEEE transactions on medical imaging, vol. 30, no. 9, pp. 1605-1616, 2011.

128. C. Mauger, S. Govil, R. Chabiniok, K. Gilbert, S. Hegde, T. Hussain, A. D. McCulloch, C. J. Occleshaw, J. Omens, J. Perry, K. Pushparajah, A. Suinesiaputra, and A. A. Young, "Right-left ventricular shape variations in tetralogy of Fallot: Associations with pulmonary regurgitation," Journal of Cardiovascular Magnetic Resonance, 2021. Accepted.

129. F. Regazzoni, D. Chapelle, and P. Moireau, "Combining Data Assimilation and Machine Learning to build data-driven models for unknown long time dynamics - Applications in cardiovascular modeling," International Journal for Numerical Methods in Biomedical Engineering, 2021. 